\title{
Foundations of dc plasma sources
}

\author{
J. T. Gudmundsson ${ }^{1,2}, *$ and A. Hecimovic ${ }^{3,4}$ \\ ${ }^{1}$ Science Institute, University of Iceland, Dunhaga 3, IS-107 Reykjavik, Iceland \\ ${ }^{2}$ Department of Space and Plasma Physics, School of Electrical Engineering, KTH-Royal Institute of Technology, \\ SE-100 44, Stockholm, Sweden \\ ${ }^{3}$ Institute for Experimental Physics II, Ruhr-University Bochum, 44801 Bochum, Germany \\ ${ }^{4}$ Max-Planck-Institut for Plasma Physics, Boltzmannstraße 2, 85748 Garching, Germany
}

(Dated: September 6, 2017)

\begin{abstract}
A typical dc discharge is configured with the negative cathode at one end and a positive anode at the other end, separated by a gas filled gap, placed inside a long glass cylinder. A few hundred volts between the cathode and anode is required to maintain the discharge. The type of discharge that is formed between the two electrodes depends upon the pressure of the working gas, the nature of the working gas, the applied voltage and the geometry of the discharge. We discuss the current-voltage characteristics of the discharge as well as the distinct structure that develops in the glow discharge region. The dc glow discharge appears in the discharge current range from $\mu \mathrm{A}$ to $\mathrm{mA}$ at $0.5-300 \mathrm{~Pa}$ pressure. We discuss the various phenomena observed in the $\mathrm{dc}$ glow discharge, including the cathode region, the positive column, and striations. The dc glow discharge is maintained by the emission of secondary electrons from the cathode target due to the bombardment of ions. For decades the dc glow discharge has been used as a sputter source. Then it is often operated as an obstructed abnormal glow discharge and the required applied voltage is in the range $2-5 \mathrm{kV}$. Typically, the cathode target (the material to be deposited) is connected to a negative voltage supply ( $\mathrm{dc}$ or $\mathrm{rf}$ ) and the substrate holder faces the target. The relatively high operating pressure, in the range from $2-4 \mathrm{~Pa}$, high applied voltages, and the necessity to have a conductive target limit the application of dc glow discharge as a sputter source. In order to lower the discharge voltage and expand the operation pressure range the lifetime of the electrons in target vicinity is increased through applying magnetic field, by adding permanent magnets behind the cathode target. This arrangement is coined the magnetron sputtering discharge. The various configurations of the magnetron sputtering discharge and its applications are described. Furthermore, the use of dc discharges for chemical analysis, the Penning discharge and the hollow cathode discharges and some of its applications are briefly discussed.
\end{abstract}

\section{INTRODUCTION}

The dc discharge is a basic and a very easily reproducible source of plasma. It is typically configured with the negative cathode at one end and a positive anode at the other end separated by a gas filled gap, sitting inside a long glass cylinder. The plasma is created by applying a dc voltage over the gas filled gap between two electrodes. Such dc discharge has been the subject of intensive research for almost 200 years. The dc discharge and in particular the de glow discharge is important historically both for studying the properties of the plasma as well as for the various applications where the dc discharge is used to provide a weakly ionized plasma.

The term gas discharge refers to the flow of electric current through a gaseous medium. If this is to occur some of the gas atoms and molecules have to be ionized. Furthermore, there has to be an electric field to drive this current. The current, which provides power to the discharge, has to be continuous throughout the length of the discharge. In the dc discharge the current is driven by both ions and electrons created within the plasma volume, as well as by electrons emitted from the cathode. Therefore, for comprehensive description of the dc dis-

*tumi@hi.is charge current, the interaction of charged particles with the electrode surfaces has to be taken into account.

The electrons and ions are charged so that application of electric or magnetic field can preferentially heat these particles while the neutral particles remain at lower temperature. A species temperature is a measure of the average energy of that species energy distribution. The various types of particles are generally not in thermal equilibrium, which means that the temperature of the different plasma species is not the same. In particular, the electrons, due to their low mass, can be easily accelerated to high energies sufficient to excite and ionize neutral atoms or molecules. Thus, the electrons have much higher temperature than the heavy particles (ions, atoms, and molecules), or $T_{\mathrm{e}} \gg T_{\mathrm{i}}, T_{\mathrm{g}}$, where $T_{\mathrm{e}}$ is the electron temperature, $T_{\mathrm{i}}$ is the ion temperature, and $T_{\mathrm{g}}$ is the neutral gas temperature.

The gas pressure is an important parameter influencing the plasma species temperature and thermal equilibrium between species. At low pressure only a few collisions occur and the energy transfer between species is inefficient. At high pressure many collisions occur between the various plasma species which results in more equal temperatures of the species. The mean free path is the average distance travelled by a particle between collisions with other particles and is reciprocal to the product of gas pressure and the collisional cross section.

The focus of this work is on presenting the fundamen- 
tal properties of the dc discharge and some of its applications. The dc discharge has numerous diverse applications, such as being a source of illumination, as gas lasers, an electron source for Hall thrusters, a pixel in a plasma display panel, and as a sputter deposition tool. Section II gives a general description of the dc discharge, through the development of the voltage-current relationship as the current increases. The axial profile of the various parameters of the dc glow discharge is discussed in section III. We discuss the electrical breakdown condition and the Paschen curve in section IV.A, the secondary electron emission from the cathode that plays a significant role in maintaining the discharge, in section IV.B, and the cathode sheath in section IV.C. The sputter process and the application of the dc discharge as a sputter source, or the diode sputter source, are discussed in section V. We discuss how and why the dc sputter source was developed into the magnetron sputtering discharge in section VI, where also the various configurations of magnetron sputtering discharges are presented. We finally discuss briefly, the application of dc discharge to chemical analysis of surfaces in section VII, the Penning discharge in section VIII and the hollow cathode discharge in section IX.

\section{THE DC DISCHARGE}

Let us assume two parallel electrodes with applied potential $V_{\mathrm{D}}$ separated by distance $L$, placed in a glass vessel. The gap between the electrodes is filled with gas at pressure $p$. The type of discharge formed between the two electrodes depends upon the (i) pressure of the working gas, (ii) the nature of the working gas, (iii) the applied voltage, and (iv) the geometry of the discharge. A discussion of the relationship between the discharge current and the applied voltage for the dc discharge can be found in review articles such as by Druyvesteyn and Penning (1940), Francis (1956) and Ingold (1978) and in a number of textbooks including those by Howatson (1976, Chapter 4), Raizer (1991, Section 8.2.), and Roth (1995, Chapter 9). The early history of discharge physics research is given in a brief review by Brown (1978), where some of the terminology used in discharge physics is explained, and some of the historical people, whose names are associated with the various phenomena observed in the dc discharges, are introduced. A typical representation of the relationship between the discharge voltage and the discharge current across a low pressure dc discharge is shown in Figure 1. The discharge is connected in series with a variable resistance $R$, that controls the current flowing through the circuit as shown by the circuit diagram in Figure 2. The discharge voltage is

$$
V_{\mathrm{D}}=V_{\mathrm{S}}-I_{\mathrm{D}} R
$$

where $V_{\mathrm{S}}$ is the voltage at the output of the voltage source and $I_{\mathrm{D}}$ is the discharge current. The curve shown in Figure 1 can be traced out by varying the load resistance $R$

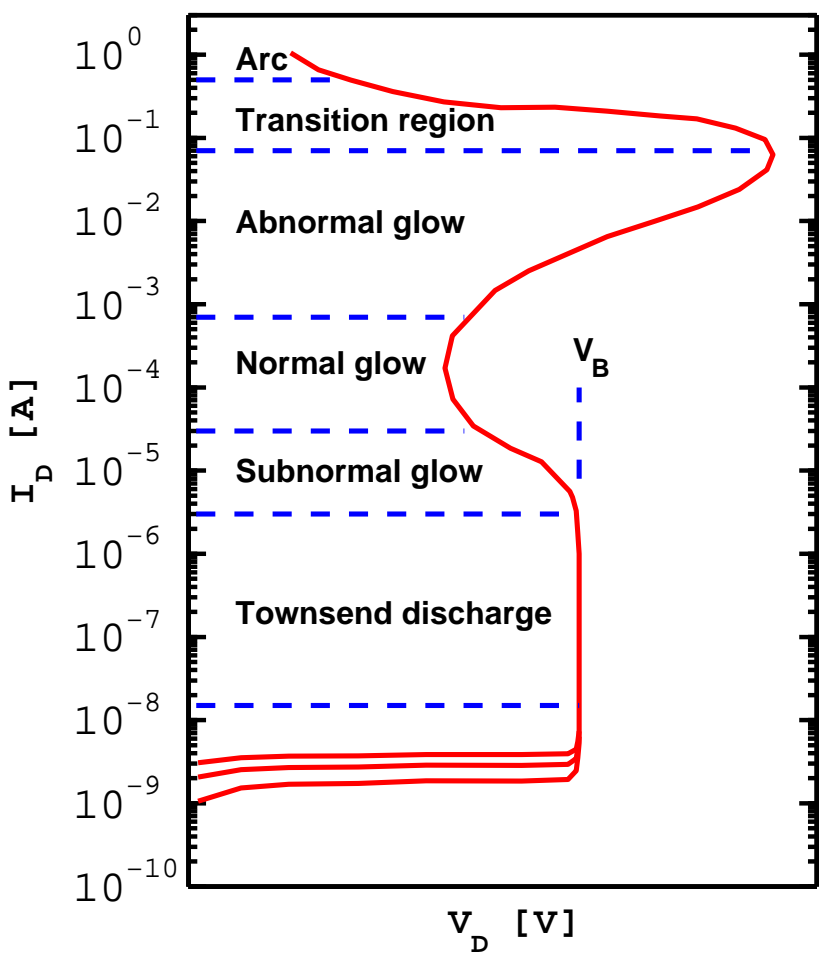

FIG. 1 The discharge current versus applied voltage for a dc discharge.

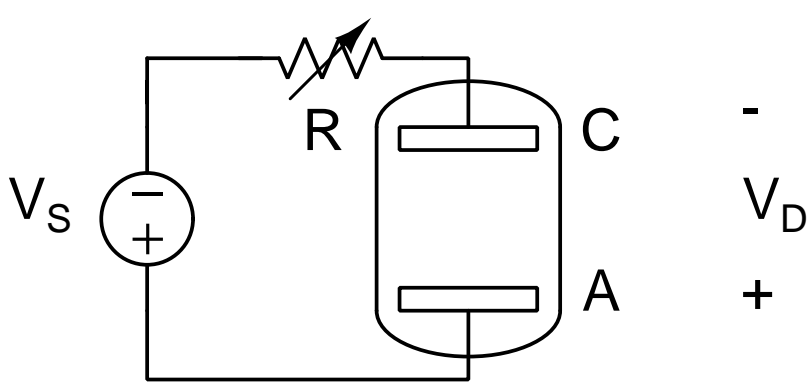

FIG. 2 A circuit diagram showing the voltage source, the variable resistor that controls the current flow through the circuit and the dc discharge.

and/or the output voltage of the source $V_{\mathrm{S}}$. When a voltage is first applied the current is very low. This current consists of contributions from various random sources such as cosmic radiation. Initially this current remains nearly constant with increased voltage. As the voltage is increased further (either by increasing $V_{\mathrm{S}}$ or lowering $R$ ) the charged particles eventually achieve enough energy to produce more charged particles though collisions with the gas atoms or bombardment of the electrodes (e.g. secondary electrons). As more charged particles are created the current increases, while the voltage is limited by the output impedance of the power supply and remains roughly constant. This region is commonly referred to 
as the Townsend discharge. The characteristics of the Townsend discharge are the very small currents and uniform electric field distribution across the entire discharge. Space charges have little influence and a sheath has not developed in the cathode vicinity. The Townsend discharge is not luminous since the electron density is low and therefore the density of excited atoms, which emit visible light, is correspondingly small. Furthermore, it is not a self-sustained discharge in the sense that it does not entirely provide its own ionization but requires some external assistance in order to produce electrons either in the gas itself or from a negative electrode. Since the current is small and the electron density is very low, the Debye length is large and comparable to the system characteristic length $L$, such that the electric field penetrates into the discharge.

If the voltage is increased further the current increases and eventually this leads to an ionization avalanche, which results in the current sharply increasing by several orders of magnitude. As the current increases an accumulation of electrons and ions occurs in front of the electrodes that deforms the field structure. By increasing the current, thus increasing the electron density, space charge distortion sets in, and the Debye length decreases and eventually a sheath, with high field strength, is formed next to the cathode. This is what is referred to as the breakdown point, and transition to the subnormal glow. It occurs at voltages ranging from two to three hundred volts upwards, depending on the nature of the gas, pressure, and the separation of the electrodes. Once a breakdown has occurred, the discharge becomes self-sustaining, the gas becomes luminous, and takes the form of a glow. The ions from the plasma are accelerated towards the negative electrode, i.e. cathode, bombarding it at energies comparable to the value of the applied voltage. As the ions bombard the electrode, secondary electrons are emitted, accelerated away from the negative cathode, gaining sufficient energy to excite and ionize the atoms of the working gas. Thus in turn more ions are available to bombard the cathode, and create more secondary electrons.

When the number of electrons generated is high enough to generate enough ions, to produce the equal number of electrons, the discharge is said to be selfsustaining. At this condition the voltage across the discharge drops and the current increases abruptly. The voltage drop indicates a more efficient ionization processes and/or reduced loss. The current is now dictated not only by the output resistance of the power supply but the discharge resistance as well. This is referred to as the normal glow or the dc glow discharge. The electron impact excitation of atoms and ions by collisions, followed by de-excitations through emission of the radiation is responsible for the characteristic glow of the normal glow. The ion bombardment is initially not uniformly distributed across the cathode surface. The discharge current arranges an optimum current density and if the current increases further, more and more of the cathode surface participates. This will continue as the supplied power is increased until a nearly uniform density is achieved covering the whole cathode area and the plsma becomes homogeneous.

In case the power is reduced at this point beyond a critical value, the transition from a homogeneous plasma to a structured plasma is observed for a dc micro-discharges in xenon, with distinct patterns emerging in the formerly homogeneous plasma layer (Schoenbach et al., 2004). The plasma pattern consist of filamentary structures arranged in concentric circles. With further reduction in current the number of filaments decreases to a minimum before the discharge extinguishes. The number of filaments and the degree of rotational symmetry depend on the gas pressure.

When the entire available cathode surface is covered by ion bombardment further increase in the applied power leads to an increase in both voltage and current. This region is referred to as the abnormal glow and is the regime used for sputtering and is discussed in section $\mathrm{V}$. The abnormal glow discharge looks much like the normal glow discharge but is more intensely luminous, and sometimes the structures near the cathode (discussed in section III) merge into one another. When increasing the current density further from the abnormal glow state the discharge transfers into arc. The kinetic energy of ions bombarding the cathode is transferred to the vibration excitation of the cathode atoms, thus heating the cathode surface. When the cathode surface gets hot enough, the electrons are emitted via thermionic emission and the discharge makes a transition into the arc regime, a plasma state characterised by low voltage and high-current.

To summarize the above discussion: dc discharges in steady state can be classified in three types according to the current which they carry. These are:

- The Townsend discharge for discharge currents up to $10^{-6} \mathrm{~A}$

- The glow discharge for discharge currents in the range $10^{-6}-10^{-1} \mathrm{~A}$

- The arc discharge for discharge currents higher than $10^{-1} \mathrm{~A}$

\section{THE DC GLOW DISCHARGE}

Over time the simplicity of the dc glow discharge geometry made it a commonly used plasma generation method for fundamental research in both discharge physics and atomic and molecular physics. The low pressure dc glow discharge has a very distinctive appearance which is shown schematically in Figure 3. Here, we will discuss these structures and the role of each region. This topic has been under investigation for almost 200 years so the literature is rather extensive. Such a discussion can be found in a number of review articles (Druyvesteyn 


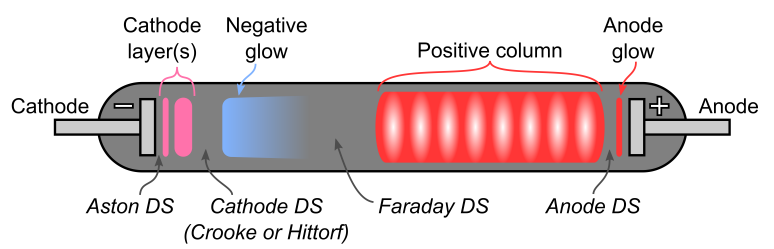

FIG. 3 A schematic of the dc glow discharge showing the several distinct regions that appear between the cathode and the anode.

and Penning, 1940; Francis, 1956; Ingold, 1978) and textbooks such as Roth (1995, Chapter 9) and Raizer (1991, Section 8.1).

As seen in Figure 1 the dc glow discharge operates the discharge current range from $\mu \mathrm{A}$ to hundreds of $\mathrm{mA}$ (current density in the range $10^{-7}-10^{-2} \mathrm{~A} / \mathrm{cm}^{2}$ ). Furthermore, the pressure is typically in the range $0.5-$ $300 \mathrm{~Pa}$. The plasma that is formed in a low pressure dc glow discharge can exhibit a structure of several distinct regions, bands of varying luminosity and color, between the cathode and the anode as seen in the schematic in Figure 3. These structures appear over a wide range of operating conditions. A photograph of the dc glow discharge showing some of the structure is shown in Figure 4.

The cathode is often made of an electrically conductive metal. The discharge is maintained by electrons emitted from the cathode as a result of positive ion bombardment of the cathode surface, referred to as secondary electron emission and discussed in section IV.B. The potential difference applied between the two electrodes is generally not equally distributed between cathode and anode. Immediately next to the cathode is the primary dark space or the Aston dark space where the secondary electrons are accumulated. This region has a strong electric field and a negative space charge, do to the accumulation of slow electrons which are in the process of being accelerated from the cathode.

Next to the Aston dark space is the cathode glow (a bright reddish or orange fluorescence at the cathode surface seen in Figure 4) with a relatively high ion density. Here the secondary electrons begin to accelerate away from the cathode to a very high velocity. These high energy electrons start to have collisions with neutral gas atoms at a distance away from the cathode corresponding to the mean-free-path, the cathode glow. The color of the light emitted is characteristic of both the cathode material and the working gas. The axial length of the cathode glow depends on the nature of the gas and gas pressure.

The cathode glow is followed by the cathode (Crookes or Hittorf) dark space where the electric field is moderate and the space charge is positive and of relatively high density. In this dark space also the positive ions are accelerated towards the cathode. Since the mobility of ions is much less than that of electrons, this dark space

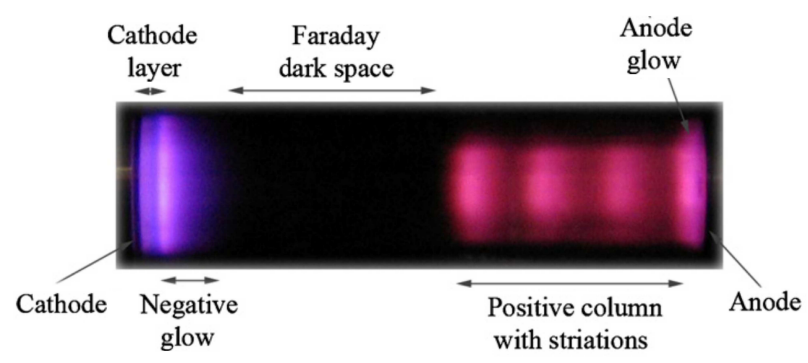

FIG. 4 The structure of a nitrogen dc glow discharge with a stratified positive column. The cathode is on the left and the anode on the right. From Lisovskiy et al. (2012).

consists mainly of ions. The cathode dark space is followed by the negative glow. The negative glow exhibits the brightest light intensity of the entire discharge (see Figure 4). The acceleration of the secondary electrons leads to excitation and ionizing collisions in this region. Here the electric field is relatively low and the current is almost entirely carried by electrons.

Figure 5 shows the spatial variations of the potential, the electric field, particle densities, space charge and current densities along the axis of a dc glow discharge. The spatial variation of the plasma parameters shown here was found by particle-in-cell simulation of an argon discharge at $50 \mathrm{~Pa}$ when a voltage of $400 \mathrm{~V}$ is applied across the $5 \mathrm{~cm}$ discharge gap (the cathode is kept at $-400 \mathrm{~V}$ ) (Budtz-Jørgensen, 2001). As the potential profile indicates (Figure 5 (a)) the electric field is large in the vicinity of the electrodes and almost zero in the plasma bulk. Thus, almost all the potential drops in the first few millimeters in front of the cathode. We see in Figure 5 (c) that the sheath region is depleted of electrons and in Figure 5 (d) we see that the net space charge is positive in the sheath region. The space charge shown in Figure 5 (d) is found by subtraction of the electron density from the ion density. In the plasma bulk the plasma is quasineutral and the electron and ion densities are the same. This space charge density leads to the electric field distribution seen in Figure 5 (b). We see from the above discussion that most of the voltage drops across the discharge appears over the cathode sheath. The cathode sheath size, the distance from the cathode surface to the boundary of the negative glow is denoted by $d_{c}$. The voltage drop is referred to as the cathode fall of $V_{\mathrm{c}}$ volts. Furthermore, most of the power dissipation in the glow discharge occurs in the cathode region. The electric field within the cathode sheath has been experimentally determined (Den Hartog et al., 1988; Doughty et al., 1987) using optogalvanic diagnostics (Doughty et al., 1985). The measurements show that the electric field exhibits a linear decrease in the cathode sheath with a gradient that depends on the current density, showing that in principle that the sheath thickness is independent of the current density for a helium discharge at $466 \mathrm{~Pa}$ (Den Hartog et al., 1988; Doughty et al., 1987). Similarly, Booth et al. 

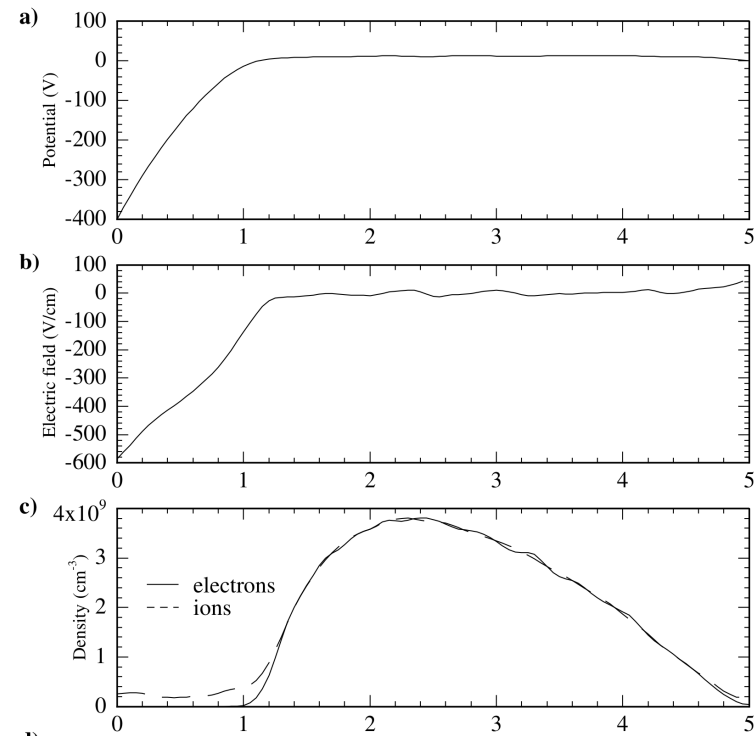

d)

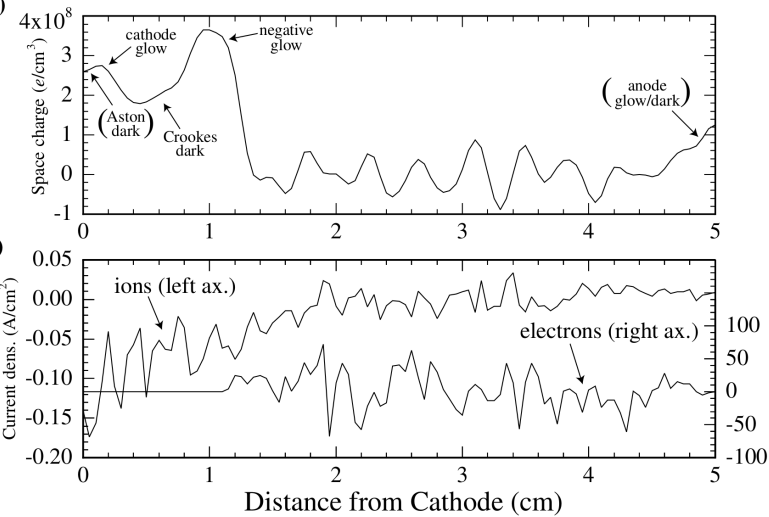

FIG. 5 Spatial profiles of (a) the plasma potential, (b) the electric field, (c) ion and electron density, (d) space-charge density, and (e) ion and electron current density. From particle-in-cell simulation of an argon discharge at $50 \mathrm{~Pa}$ and -400 V applied to the cathode. From Budtz-Jørgensen (2001).

(1994) applied a laser-induced fluoresence (LIF) to measure a roughly linear decrease in the electric field strength as a function of distance from the cathode in hydrogen discharge. They see decreasing sheath thickness with increasing neutral gas pressure.

The negative glow is followed by the Faraday dark space where the electric field is low as well as the energy of electrons. The electrons do not have enough energy to excite the atoms or molecules of the working gas and electrons recombine with ions. Thus this appears as a dark region in Figure 4. The Faraday dark space is followed by the positive column. The positive column is a quasineutral plasma where the electric field is very low. The positive column appears as a long uniform glow, except when striations are formed (in Figure 4 striations appear in the positive column). The positive column is a quasineutral plasma that acts as a conducting path between negative glow region and the anode and will be discussed
TABLE I The color of selected luminous zones in the dc glow discharge. Based on Francis (1956).

\begin{tabular}{clll}
\hline \hline Gas & $\begin{array}{l}\text { Cathode } \\
\text { layers }\end{array}$ & $\begin{array}{l}\text { Negative } \\
\text { glow }\end{array}$ & $\begin{array}{l}\text { Positive } \\
\text { column }\end{array}$
\end{tabular}

$\begin{array}{llll}\mathrm{He} & \text { red } & \text { pink } & \text { red/pink } \\ \mathrm{Ne} & \text { yellow } & \text { orange } & \text { red/brown } \\ \mathrm{Ar} & \text { pink } & \text { dark blue } & \text { dark red } \\ \mathrm{H}_{2} & \text { red/brown } & \text { pale blue } & \text { pink } \\ \mathrm{O}_{2} & \text { red } & \text { yellowish/white } & \text { pale yellow/pink center } \\ \mathrm{N}_{2} & \text { pink } & \text { blue } & \text { red/yellow } \\ \mathrm{Air} & \text { pink } & \text { blue } & \text { red/yellow }\end{array}$

further below (section III.A). Often a thin dark space is observed at the end of the positive column and a glow at the anode surface. The anode dark space has a negative space charge due to electrons that flow from the positive column to the anode. Close to the anode, electrons are attracted and accelerated, but ions are repelled. The accelerated electrons excite atoms or molecules, so the anode glow is a bright region that appears at the anode (see Figure 4). The drift velocity of the electrons in the positive column is low as the electric field is weak and typically less than the electrons thermal velocity. This calls for a retarding electric field in front of the anode to prevent the full thermal electron current from reaching the anode. Recall that the anode must be positive with respect to the positive column in order to maintain the current. This leads to formation of a double layer. But the voltage drop in this region is small and plays little role in the overall discharge dynamics.

The size, intensity and color of all the regions described above are dependent on the working gas, gas pressure, and applied voltage. Also some of the features may be absent over particular parameter ranges. The various gases exhibit a discharge of a characteristic colour. The colors of the light emitted from the various zones of the dc glow discharge are listed in Table I. If the pressure is reduced the cathode dark space expands at the expense of the positive column. This is due the fact that now the electrons have to travel farther (mean free path is longer) to produce efficient ionization. For a secondary electron emission yield in the range $0.05-0.1$ each secondary electron must produce roughly $10-20$ ions to maintain the glow discharge. The discharge current at the cathode consists of electron current $I_{\mathrm{e}}$ and ion current $I_{\mathrm{i}}$, or

$$
I_{\mathrm{D}}=I_{\mathrm{e}}+I_{\mathrm{i}}=I_{\mathrm{i}}\left(1+\gamma_{\mathrm{see}}\right)
$$

where $\gamma_{\text {see }}$ is the secondary electron emission yield, so at the target, the dominating fraction of the discharge current is due to ions. Thus at the cathode most of the current is carried by ions flowing towards the cathode while 
in other regions of the discharge including the negative glow and the Faraday dark space nearly all the current is carried by electrons. This shows that the ionization processes in the cathode dark space are essential for the maintenance of the discharge. Den Hartog et al. (1988) determined experimentally the ratio of ion to electron current at the cathode, to be 3.3 for helium discharge at $466 \mathrm{~Pa}$ showing almost no dependence on the total discharge current. Furthermore, they found that the majority of ions hitting the cathode are produced within the cathode sheath, and that the discharge current is almost solely carried by beam electrons at the boundary between the cathode sheath and the negative glow. How many ions each secondary electron produces depends on its mean free path and the distance between the anode and cathode. This relation is qualitatively the statement of Paschen's law that relates the breakdown voltage $V_{\mathrm{B}}$ to the product of gas pressure and electrode separation and will be discussed in section IV.A.

\section{A. The positive column}

If the length of the discharge tube is multiple times the radius of the tube, then most of the tube is filled with what appears to be a glow of uniform light intensity. This is the positive column. The positive column constitutes a quasi neutral plasma. Its sole role is to maintain conduction of current by electrons which is established in the cathode vicinity. When a typical dc glow discharge is operated at a fixed pressure and the length is reduced, the length reduction is taken up entirely by the positive column, and the other regimes remain unchanged. This continues until the anode has reached the negative glow, at which point the voltage required to maintain the discharge begins to rise.

The positive column is a weakly ionized plasma often with cylindrical geometry, bounded by non-conducting walls and sustained by an axial current. The positive column is axially uniform and azimuthally symmetric and the various parameters vary only radially. Its properties are independent of the length of the column and it can be exptended infinetely. As the charged particles are lost to the tube walls the discharge is not radially uniform. The positive column is luminous as the electrons have energy high enough to ionize atoms and thus to excite atoms. The color of the positive column is characteristic of the gas and is generally not the same as that of the negative glow, nor is it as luminous. The electron or ion density is typically in the range $10^{15}-10^{16} \mathrm{~m}^{-3}$ within the positive column. The potential decreases radially and thus confines the electrons while driving the ions outwards towards the walls. At steady state there is a balance between the electron-ion pairs created via electron impact ionization in the bulk and the electron-ion pairs lost to the walls. Within the positive column the only ionization that takes place is to compensate for losses do to recombination and diffusion to the chamber walls. The relative importance of these processes depends on the geometry of the discharge, gas pressure, and the current flow.

Most of the theoretical treatments of the positive column have been based on the classical ambipolar theory of Schottky (1924) and the free fall theory of Tonks and Langmuir (1929). This is how the positive column is often described in review articles (Ingold, 1978), and in text books for pedagogical purposes including Roth (1995, Section 9.2.), Cherrington (1979, Chapter 8), and Lieberman and Lichtenberg (2005, Section 14.2.). In this treatment, referred to as the local approach, the electron energy distribution is often assumed to be Maxwellian, the Boltzmann equation is not solved, and that one-step ionization of the working gas is the sole electron source. The electrons are assumed to be in equilibrium with the axial electric field and the average electron energy is radially invariant in this assumption. Within the positive column the quantities such as the electric field and electron and ion densities are assumed to be independent of the longitudinal position and depend only on the radial distance from the tube walls. Hence, there is a balance between the production of charged particles by ionizing collisions of energetic electrons with neutrals and loss of charged particles by diffusion towards the tube walls where they recombine. This can be written as a particle balance

$$
-\nabla \cdot D_{\mathrm{a}} \nabla n=\nu_{\mathrm{iz}} n
$$

where $n=n_{\mathrm{e}}=n_{\mathrm{i}}$ is the plasma density, $D_{\mathrm{a}}$ is the ambipolar diffusion coefficient, $\nu_{\mathrm{iz}}=k_{\mathrm{iz}} n_{\mathrm{g}}$ is the electron impact ionization rate, and $k_{\mathrm{iz}}$ is the electron impact ionization rate coefficient. The electron impact ionization rate coefficient can be written

$$
k_{\mathrm{iz}}=\sigma_{0} \bar{v}_{\mathrm{e}}\left(1+\frac{2 \mathrm{~T}_{\mathrm{e}}}{\mathcal{E}_{\mathrm{iz}}}\right) \exp \left(-\frac{\mathcal{E}_{\mathrm{iz}}}{\mathrm{T}_{\mathrm{e}}}\right)
$$

where $\sigma_{0}=\pi\left(e / 4 \pi \epsilon_{0} \mathcal{E}_{\mathrm{iz}}\right)^{2}, \mathcal{E}_{\mathrm{iz}}$ is the ionization potential, and we have assumed a Thomson cross section that is expanded near $\mathcal{E}=\mathcal{E}_{\mathrm{iz}}$, and $\bar{v}_{\mathrm{e}}=\left(8 e \mathrm{~T}_{\mathrm{e}} / \pi m\right)^{1 / 2}$ is the mean speed of electrons with a Maxwellian velocity distribution at temperature $\mathrm{T}_{\mathrm{e}}$ (Lieberman and Lichtenberg, 2005, p. 71 and 79). Thus we can use the term electron temperature to describe the average energy of the electron gas. Here we use the roman typeface symbol $\mathrm{T}$ for the voltage equivalent of the temperature.

When the flux of electrons and ions to an insulating wall is equal the plasma has achieved a steady state. As the electrons are significantly lighter than ions, they have much higher mobility, so that an electric field is created to maintain a local flux balance. The diffusion coefficient that describes the ion and electron diffusion to the tube walls in steady state, is referred to as the ambipolar diffusion coefficient (see Lieberman and Lichtenberg (2005, p. 135-136) or Roth (1995, p. 157-158)). When the mobilty of electrons is much larger than the mobilty of ions we find that $D_{\mathrm{a}} \approx D_{\mathrm{i}}\left(1+\mathrm{T}_{\mathrm{e}} / \mathrm{T}_{\mathrm{i}}\right)$ where $D_{\mathrm{i}}=e \mathrm{~T}_{\mathrm{i}} / M_{\mathrm{i}} \nu_{\mathrm{mi}}$ is the ion diffusion coefficient, $M_{\mathrm{i}}$ is the ion mass, $\nu_{\mathrm{mi}}=n_{\mathrm{g}} k_{\mathrm{mi}}$ is the ion momentum trans- 
fer frequency, and $k_{\mathrm{mi}}$ isthe ion momentum transfer rate coefficient. In general $k_{\mathrm{mi}}$ depends on the gas temperature $T_{\mathrm{g}}$ and the ion temperature $T_{\mathrm{i}}$. For $\mathrm{T}_{\mathrm{e}} \gg \mathrm{T}_{\mathrm{i}}$ we see that $D_{\mathrm{a}} \approx e \mathrm{~T}_{\mathrm{e}} / M_{\mathrm{i}} \nu_{\mathrm{mi}}$ and the ambipolar diffusion coefficient is much greater than the diffusion coefficient for the ions. Following Schottky (1924) and Cobine (1958, section 8.11 ) the ion particle balance equation can be written in cylindrical coordinates

$$
\frac{\mathrm{d}^{2} n}{\mathrm{~d} r^{2}}+\frac{1}{r} \frac{\mathrm{d} n}{\mathrm{~d} r}+\frac{\nu_{\mathrm{iz}}}{D_{\mathrm{a}}} n=0
$$

which is Bessel's equation. The solution of Bessel's equation is

$$
n(r)=n_{0} \mathrm{~J}_{0}\left(r \sqrt{\frac{\nu_{\mathrm{iz}}}{D_{\mathrm{a}}}}\right)
$$

where $\mathrm{J}_{0}$ is a Bessel function of zero order and $n_{0}$ is the plasma density on the discharge axis, which will be determined later in terms of the discharge current. If the ion-neutral mean free path and the sheath thickness are both small compared to the column (tube) radius, or $\lambda_{\mathrm{i}}, s \ll R$, then $n(R) \approx 0$ and we have

$$
\sqrt{\frac{\nu_{\mathrm{iz}}}{D_{\mathrm{a}}}}=\frac{\chi_{01}}{R}
$$

where $\chi_{01} \approx 2.405$ is the first zero of the Bessel function of zero order. Both the ionization rate $\nu_{\mathrm{iz}}$ and the ambipolar diffusion coefficient $D_{\text {a }}$ depend on the electron temperature. If Eq. (4) is substituted into Eq. (7) we find that

$$
\frac{\sigma_{0} \bar{v}_{\mathrm{e}}\left(1+\frac{2 \mathrm{~T}_{\mathrm{e}}}{\mathcal{E}_{\mathrm{iz}}}\right)}{e \mathrm{~T}_{\mathrm{e}} / M_{\mathrm{i}} k_{\mathrm{mi}}} \exp \left(-\frac{\mathcal{E}_{\mathrm{iz}}}{\mathrm{T}_{\mathrm{e}}}\right)=\left(\frac{\chi_{01}}{R n_{\mathrm{g}}}\right)^{2}
$$

which can be solved for $\mathrm{T}_{\mathrm{e}}$. This can be approximated, given $\mathrm{T}_{\mathrm{e}} \ll \mathcal{E}_{\text {iz }}$ and $v_{\mathrm{e}} \propto \sqrt{\mathrm{T}_{\mathrm{e}}}$, as

$$
\frac{\exp \left(\mathcal{E}_{\mathrm{iz}} / \mathrm{T}_{\mathrm{e}}\right)}{\sqrt{\mathrm{T}_{\mathrm{e}}}} \propto(R p)^{2}
$$

where the constant of proportionality depends on the type of gas. So for a given gas (given $\mathcal{E}_{\mathrm{iz}}$ and $M_{\mathrm{i}}$ ) the electron temperature is a unique function of the pressure radius product and is independent of the plasma density or discharge current. This relation is valid only when the electron mean free path is small compared to the tube radius.

The longitudinal electric field $E$ is required to maintain the current in the presence of the diffusion losses. To determine the electric field we apply the power balance. The ohmic power absorbed is given by

$$
P_{\text {abs }}=2 \pi \int_{0}^{R} \mathbf{J} \cdot \mathbf{E} r \mathrm{~d} r
$$

and the power lost due to the ion loss at the tube walls

$$
P_{\text {loss }}=2 \pi R \Gamma_{r} e \mathcal{E}_{\mathrm{T}}
$$

where $e \mathcal{E}_{\mathrm{T}}=e\left(\mathcal{E}_{\mathrm{c}}+\mathcal{E}_{\mathrm{i}}+\mathcal{E}_{\mathrm{e}}\right)$ is the total energy lost per electron-ion pair created, and $\mathcal{E}_{\mathrm{i}}+\mathcal{E}_{\mathrm{e}}$ is the kinetic energy lost to the wall per electron-ion pair lost to the wall. For a Maxwellian electron energy distribution and an insulating wall $\mathcal{E}_{\mathrm{i}}+\mathcal{E}_{\mathrm{e}}=\frac{1}{2} \mathrm{~T}_{\mathrm{e}} \ln \left(M_{\mathrm{i}} / 2 \pi m_{\mathrm{e}}\right)+2 \mathrm{~T}_{\mathrm{e}}$. $\mathcal{E}_{\mathrm{c}}$ is the collisional loss per electron-ion pair created

$$
\mathcal{E}_{\mathrm{c}}=\mathcal{E}_{\mathrm{iz}}+\sum_{i} \mathcal{E}_{\mathrm{ex}, i} \frac{k_{\mathrm{ex}, i}}{k_{\mathrm{iz}}}+\frac{k_{\mathrm{el}}}{k_{\mathrm{iz}}} \frac{3 m_{\mathrm{e}}}{M_{\mathrm{i}}} T_{\mathrm{e}}
$$

where $\mathcal{E}_{\text {iz }}$ is the ionization energy, $\mathcal{E}_{\text {ex }, i}$ and $k_{\text {ex }, i}$ are the excitation energy and rate coefficient for the $i$-th excitation process, respectively, $k_{\mathrm{el}}$ is the elastic scattering rate coefficient. The electric field along the column can be determined by equating the absorbed and lost power and substituting the radial density solution (Eq. (6)) we have

$$
e n_{0} \mu_{\mathrm{e}} E^{2} 2 \pi \int_{0}^{R} \mathrm{~J}_{0}\left(r \sqrt{\frac{\nu_{\mathrm{iz}}}{D_{\mathrm{a}}}}\right) r \mathrm{~d} r=2 \pi R\left(D_{\mathrm{a}} \nu_{\mathrm{iz}}\right)^{1 / 2} n_{0} \mathrm{~J}_{1}\left(R \sqrt{\frac{\nu_{\mathrm{iz}}}{D_{\mathrm{a}}}}\right) e \mathcal{E}_{\mathrm{T}}
$$

where a constant mobility $\mu_{\mathrm{e}}=e / m_{\mathrm{e}} \nu_{\mathrm{m}}$, and constant electric field are assumed, where $m_{\mathrm{e}}$ is the electron mass, $\nu_{\mathrm{m}}=k_{\mathrm{m}} n_{\mathrm{g}}$ is the electron neutral collision frequency, $k_{\mathrm{m}}$ is the electron neutral collision rate coefficient (depends on $\mathrm{T}_{\mathrm{e}}$ ), and the current density

$$
J=e n \mu_{\mathrm{e}} E
$$

has been substituted. Performing the integration and solve for $E$ gives

$$
E=\left(\frac{\nu_{\mathrm{iz}} \mathcal{E}_{\mathrm{T}}}{\mu_{\mathrm{e}}}\right)^{1 / 2}=\left(\frac{m_{\mathrm{e}}}{e} \nu_{\mathrm{iz}} \nu_{\mathrm{m}} \mathcal{E}_{\mathrm{T}}\right)^{1 / 2}
$$

where the electron mobility $\mu_{\mathrm{e}}$ has been substituted. This 
can be written as

$$
\frac{E}{n_{\mathrm{g}}}=\left(\frac{m_{\mathrm{e}}}{e} k_{\mathrm{iz}} k_{\mathrm{m}} \mathcal{E}_{\mathrm{T}}\right)^{1 / 2}
$$

where the right hand side only depends on the type of gas and the electron temperature. For a fixed pressureradius product, the longitudinal electric field, and hence the voltage drop along a discharge, is fixed and is independent of the discharge current. Even though the analysis is somewhat oversimplified, this conclusion is mostly valid for many diffusion controlled positive column discharges. We can eliminate $\nu_{\text {iz }}$ using Eq. (7) to obtain

$$
E=\frac{\chi_{01}}{R}\left(\frac{D_{\mathrm{a}} \mathcal{E}_{\mathrm{T}}}{\mu_{\mathrm{e}}}\right)^{1 / 2}=\frac{\chi_{01}}{R}\left(\frac{m k_{\mathrm{m}}}{M_{\mathrm{i}} k_{\mathrm{mi}}} \mathcal{E}_{\mathrm{T}} \mathrm{T}_{\mathrm{e}}\right)^{1 / 2}
$$

where we have also substituted the ambipolar diffusion coefficient. Eq. (17) indicates that the electric field depends only on $\mathrm{T}_{\mathrm{e}}$ and is independent of the neutral gas pressure. As noted above we need to determine the axial plasma density $n_{0}$. Integrating Eq. (14) over the discharge cross section then gives the current

$$
I=2 \pi e n_{0}\left(\frac{R^{2}}{\chi_{01}}\right) \mathrm{J}_{1}\left(\chi_{01}\right) \mu_{\mathrm{e}} E
$$

which for a given discharge current and the electric field given by Eq. (17) gives the axial plasma density $n_{0}$. We note that Eq. (16) and Eq. (17) suggest that the electric field is independent of the discharge current, whereas measurements usually show that the electric field $E$ decreases with increasing discharge current (Ingold, 1978). This is called the negative voltage-current curve and it has been related to significant and increasing energy transfer from electrons to neutrals via elastic collisions as the current density is increased. Thus the gas temperature is higher and the neutral gas density lower in the discharge center than at the tube wall, leading to higher electron mobilty in the discharge center (Ecker and Zöller, 1964). Another mechanism that could lead to a negative voltage-current curve in the positive column is multistep ionization.

In reality, the electron energy distribution is not Maxwellian, there are higher average electron energies near the column edge, one step ionization is typically not the only path for ionization, and there is a significant radial variation in the average energy of the electrons in these discharges (Uhrlandt and Winkler, 1996). The local approach is valid only for small values of $p L$, where the electron energy distribution is close to being Maxwellian.

For higher values of $p L$, the average electron energy can be significantly higher near the plasma edge than in the discharge center. This is because the colder electrons are confined by the potential in the discharge center while the higher energy electrons can overcome the potential energy hill. However, a full kinetic treatment, including the radial density variation is rather complicated. Instead various approximate kinetic methods have been employed to explore the positive column. One such method is the non-local approximation, originally suggested by Bernstein and Holstein (1954). The non-local approximation is an efficient method for the solution of the spatially dependent Boltzmann equation, but it is valid only in the limiting case that the energy relaxation length of electrons exceeds the discharge dimensions (Bernstein and Holstein, 1954; Busch and Kortshagen, 1995; Tsendin, 1995). This indicates that the pressure has to be low. This method assumes that the total electron energy can be assumed constant and the entire electron kinetics can be described by a single, spatially homogeneous distribution of total electron energy which is determined from a spatially averaged kinetic equation. Then the average energy, transport coefficients and collision frequencies can vary radially across the positive column even though the axial field does not. The non-local moment method is described by Ingold (1997) and compared to other approaches that can be extended to higher pressure regimes. They also state that the properties of the positive column at any pressure can be described adequately by moment equations, if the radial heat flow in the electron gas is taken into account, and when the electron transport coefficients and the relevant collision frequencies are allowed to depend on the radially varying average energy. Particle-in-cell simulation of the positive column show that the radial electron heat flow plays a major role in maintaining the global power balance in low pressure positive column discharge (Kawamura and Ingold, 2001).

A tutorial on fluid modelling of the positive column of dc glow discharges was recently given by Alves (2007). There it is described how the electron transport parameters and rate coefficients are calculated using a local mean energy approximation along with a two-term Boltzmann solver. The obtained results allow to study the discharge energy deposition features in radial direction, as a function of pressure and axial current, along with the analysis of the space-charge sheath formation. The results show that the electron mean energy is almost constant in the plasma bulk, exhibiting a strong decrease near the discharge wall, following the electron density profile. The power balance in the bulk is dominated by Joule heating and by collisions across the discharge, while in the sheath, the power is balanced by the power lost in maintaining the space-charge field and the power gained from convection.

\section{B. Striations}

The positive column sometimes shows distinct and regular luminous bands along its length, that can be either moving or stationary, referred to as striations (Kolobov, 2006; Pekarek, 1968; Raizer, 1991). Stratification of the positive column in a dc glow discharges into alternating bright and dark areas is a well-studied phenomenon, first time observed by Michael Faraday in the 1830s. These 


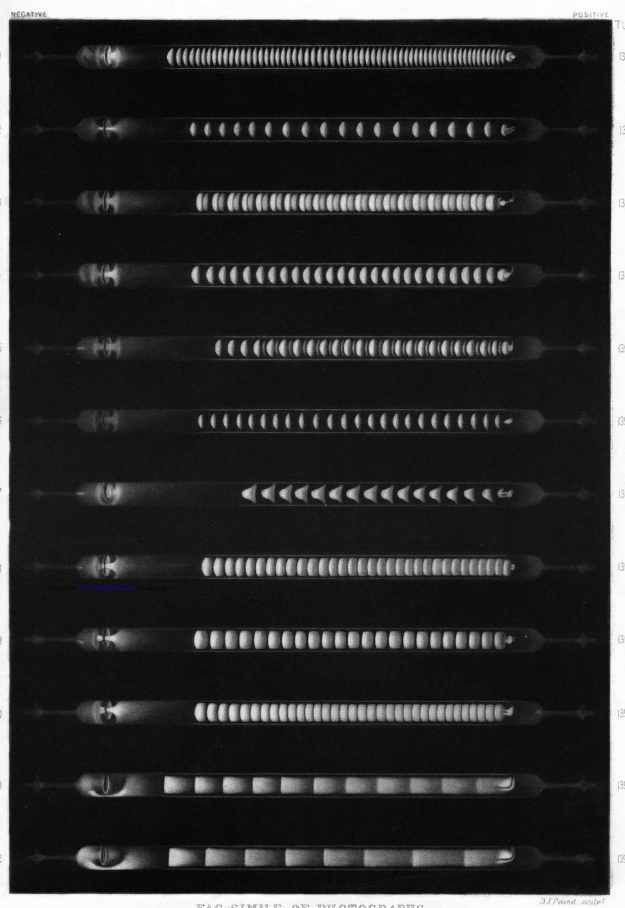

FIG. 6 Photograph of standing striations in a low pressure dc glow discharge tube. The cathode is on the left and the anode is on the right. From de la Rue and Müller (1878).

are due to fluctuations in the electron and ion density. The striation consists of a bright region, with a predominant generation of charged particles due to ionization, and a dark region were particle loss due to ambipolar escape to the discharge tube walls, and attachment to gas molecules and recombination in electronegative gas, is predominant. For the operation of the discharge at low pressure the stratified state is energetically favorable, because for a given discharge current the voltage drop and the power dissipated in the positive column when striations are present are lower than in the uniform discharge (Raizer, 1991, Section 9.7).

The standing striations can be observed by the naked eye in molecular gases (such as hydrogen, nitrogen, oxygen) and in mixtures of molecular and rare gases (Lisovskiy et al., 2012). Such stationary striations can be seen in Figures 4 and 6 . In pure rare gases, standing striations exist in the form of damping oscillations in front of cathode. Moving striations are travelling waves in the electron density. The moving striations typically exhibit frequencies in the range from a few $\mathrm{Hz}$ up to several tens of kHz. Moving striations cannot be observed by the naked eye due to their large velocity and require phase sensitive stroboscopic methods to be observed. In molecular gases they usually move from cathode towards anode. Moving striations are discussed by Oleson and Cooper (1968).

Striations can be observed in a limited range of current values, gas type, gas pressure, and tube radius. Garscadden (1978) discusses the ranges of occurrence for various gases and operating conditions. Moving striations occur at low pressures, where the ionization mean free paths are long, longer or comparable to the density gradient scale length of the gas. Thus Fick's law may not be valid. Watanabe and Oleson (1955) include both continuity and momentum equations for ions and electrons and the ion and electron densities are coupled through Poisson's equation. They demonstrate propagating ion density waves with phase velocity directed towards the cathode. Pekárek and Krejčí (1962) under the assumption of ambipolar diffusion use both the continuity and momentum equations along with Poissions equation and a simple energy balance equation. These analyses are summarized by Garscadden (1978).

\section{THE OPERATION OF THE DC GLOW DISCHARGE}

In the above discussion we have used some terms like electrical breakdown and secondary electron emission. These phenomena are important in discharge physics and in particular for the operation of the dc discharge and will be explained briefly.

\section{A. Vacuum breakdown}

Electrical breakdown is an important phenomenon in discharge physics. Electrical breakdown is the process that describes the transition from a neutral gas to a selfsustained discharge. Thus, with the application of an electric field an electrically insulating gas transitions to a conducting state. The breakdown process is highly transient and a good time resolution imagning is required to study it. This kind of studies have been performed by Wagenaars et al. $(2005,2006)$ who apply time reolved intensified charge-coupled device (ICCD) imaging to explore the breakdown process in low-pressure discharges, as well as direct measurements of the electric field strengths in ionization fronts during breakdown (Wagenaars et al., 2007). They record a formation of a light emission region in front of the anode and then movement of this light front from the anode to the cathode as breakdown is initiated. The time scales involved in the breakdown process are mainly determined by the ion drift velocity (Wagenaars et al., 2005). This observation they explain by a continuous electron avalanche developing in the discharge gap due to secondary electron emission from the cathode. This avalanche causes light emission due to excitation of the discharge gas and modification of the potential in the electrode gap due to a build-up of space charge. They conclude that the breakdown process is controlled by the development of multiple electron avalanches consistent with the standard view of Townsend breakdown into a glow discharge at low pressures. Here we derive the breakdown voltage as a func- 
tion of the product $p L$, the reduced electrode distance, the so called Paschen curve. Similar discussion can be found in various textbooks such as by Lieberman and Lichtenberg (2005, pg. 457-463), Raizer (1991, Chapter 7) and Roth (1995, Section 8.6).

The electron density and flux grow exponentially from the cathode. Thus the increase in the electron flux is proportional to the electron flux or

$$
\frac{\mathrm{d} \Gamma_{\mathrm{e}}}{\mathrm{d} z}=\alpha \Gamma_{\mathrm{e}}
$$

where $\Gamma_{\mathrm{e}}$ is the electron flux and $\alpha$, commonly expressing the number of ionisation collisions per unit length by an electron or $\alpha(z) \equiv 1 / \lambda_{\mathrm{iz}}$, the inverse of the mean free path for ionization, and is known as Townsend's first ionization coefficient. The first Townsend coefficient or ionization coefficient is a complicated function of the accelerating field and gas pressure. The electron flux in the direction along the discharge axis (or the direction of the electric field) is then

$$
\Gamma_{\mathrm{e}}(z)=\Gamma_{\mathrm{e}}(0) \exp \left[\int_{0}^{z} \alpha\left(z^{\prime}\right) \mathrm{d} z^{\prime}\right] .
$$

Due to continuity of the total charge (creation of equal numbers of electron - ion pairs) we can write

$$
\Gamma_{\mathrm{i}}(0)-\Gamma_{\mathrm{i}}(L)=\Gamma_{\mathrm{e}}(0)\left\{\exp \left[\int_{0}^{L} \alpha\left(z^{\prime}\right) \mathrm{d} z^{\prime}\right]-1\right\}
$$

where $\Gamma_{\mathrm{e}}(L)$ from Eq. (20) has been inserted. Since the discharge must be self sustaining we have $\Gamma_{e}(0)=$ $\gamma_{\text {see }} \Gamma_{i}(0)$ and $\Gamma_{\mathrm{i}}(L)=0$. Then

$$
\exp \left[\int_{0}^{L} \alpha\left(z^{\prime}\right) \mathrm{d} z^{\prime}\right]=1+\frac{1}{\gamma_{\text {see }}}
$$

is the condition for self-sustainability. In a vacuum region, the electric field $E$ is a constant and it follows that the electron drift velocity $\mu_{\mathrm{e}} E$ is also a constant. Hence the electron energy $\mathcal{E}_{\mathrm{e}}$ is a constant, allowing us to treat $\alpha$ as a constant in Eq. (22). Taking the logarithm of both sides gives

$$
\alpha L=\ln \left(1+\frac{1}{\gamma_{\text {see }}}\right)
$$

which is the breakdown condition for a dc discharge. The ionization coefficient is usually expressed in the form

$$
\frac{\alpha}{p}=A \exp \left(-\frac{B p}{E}\right)
$$

where $A$ and $B$ are determined experimentally and found to be roughly constant over a range of pressures and fields for a given gas type. The coefficients $A$ and $B$ for various common gases are listed in Table II.
TABLE II Constants for the Townsend ionization coefficient. The data is based on Lieberman and Lichtenberg (2005).

\begin{tabular}{cccc}
\hline \hline Gas & $\begin{array}{c}\mathrm{A} \\
{\left[\mathrm{cm}^{-1} \mathrm{~Pa}^{-1}\right]}\end{array}[\mathrm{V} /(\mathrm{cm} \mathrm{Pa})]$ & $\begin{array}{c}\text { Range of } E / p \\
{[\mathrm{~V} /(\mathrm{cm} \mathrm{Pa})]}\end{array}$ \\
\hline & & & \\
$\mathrm{H}_{2}$ & 0.0360 & 1.0201 & $1.1251-3.0004$ \\
$\mathrm{He}$ & 0.0210 & 0.5776 & $0.2250-1.8752$ \\
$\mathrm{Ar}$ & 0.0862 & 1.3202 & $0.7501-4.5006$ \\
$\mathrm{O}_{2}$ & 0.0488 & 1.4402 & $0.3750-0.9751$ \\
$\mathrm{~N}_{2}$ & 0.0885 & 2.4378 & $0.7501-4.5006$
\end{tabular}

If the minimum voltage of which the discharge initiates, the breakdown voltage, is written $V_{\mathrm{B}}=E L$ then

$$
A p L \exp \left(-\frac{B p L}{V_{\mathrm{B}}}\right)=\ln \left(1+\frac{1}{\gamma_{\mathrm{see}}}\right)
$$

which we solve for $V_{\mathrm{B}}$ to get

$$
V_{\mathrm{B}}=\frac{B p L}{\ln (A p L)-\ln \left[\ln \left(1+1 / \gamma_{\text {see }}\right)\right]}
$$

which is a function of the product $p L$. The curve that shows $V_{\mathrm{B}}$ as a a function of the product $p L$ is called the Paschen curve and is shown for a few common gases in figure 7. This curve shows that for a fixed discharge length $L$ there is an optimum pressure for plasma breakdown. The Paschen curve is unique to each gas or gas mixture. At the lower pressures the ionization process is ineffective due to the low electron-neutral collision probability while at higher pressures elastic collisions prevent the electrons from reaching high enough energy for ionization to occur. This behavior was first reported by de La Rue and Müller (1880) for discharges in hydrogen and air. The Paschen curve has been measured for dc discharges of various gases including nitrogen (Lisovskiy et al., 2000; Miller, 1963), argon (Lisovskiy et al., 2000), carbon tetrafluoride $\left(\mathrm{CF}_{4}\right)$ (Lisovskiy et al., 2015), and water vapour (Sivoš et al., 2015; Škoro et al., 2011).

In fact the product of pressure and distance between the electrodes $(p L)$ is the most suitable parameter to characterize the discharge. For instance, at lower pressure, the distance between cathode and anode has to be longer to create a discharge with properties comparable to those of high pressure with small distance between the electrodes. The number of gas atoms or molecules in the space between the electrodes is proportional to $p L$. For low pressure the electron mean free path is large, and most electrons reach the anode without colliding with gas atoms or molecules. Thus, at low the pressure the higher the value of $V_{\mathrm{B}}$ is required to generate enough electrons to cause the breakdown of the gas. At higher pressures the electron mean free path is short. The electrons do not gain enough energy from the electric field to ionize the gas atoms or molecules due to their frequent colli- 


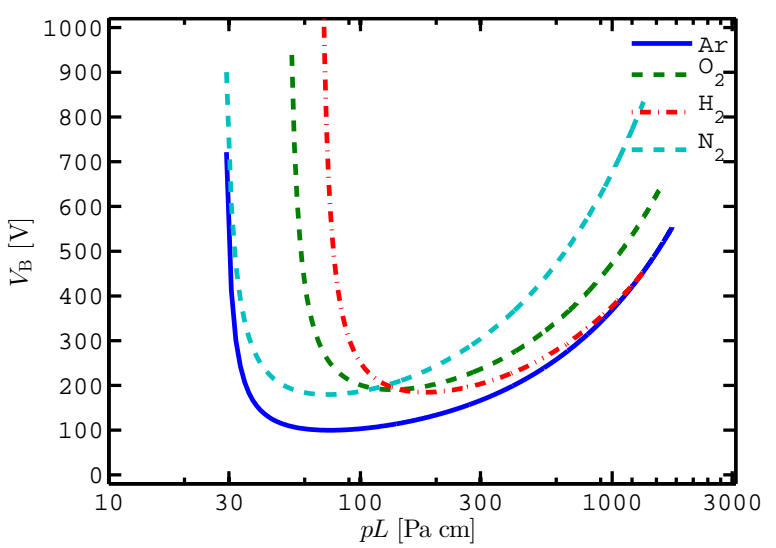

FIG. 7 The breakdown voltage $V_{\mathrm{B}}$ versus the reduced electrode distance $p L$ for a few common gases calculated using Eq. (26) and the data given in Table II, assuming $\gamma_{\text {see }}=0.1$.

sions with the gas molecules. Therefore $V_{\mathrm{B}}$ increases as the pressure increases. To find the value of $p L$ that corresponds to a minimum breakdown voltage we differentiate the expression for the breakdown voltage, Eq. (26) with respect to $p L$ and set the derivative equal to zero (Raizer, 1991, Chapter 7)

$$
(p L)_{\min }=\frac{\exp (1)}{A} \ln \left(1+\frac{1}{\gamma_{\text {see }}}\right)
$$

and the minimum voltage is the

$$
V_{\mathrm{B}, \min }=\exp (1) \frac{B}{A} \ln \left(1+\frac{1}{\gamma_{\text {see }}}\right)
$$

and is referred to as the minimum sparking potential, and is the minimum voltage at which electrical breakdown can occur in a given gas. A low pressure de glow discharge will adjust the length of the cathode region such that a minimum value of the product $p d_{\mathrm{c}}$ is established

$$
p d_{\mathrm{c}} \approx(p L)_{\min }
$$

which is the Paschen minimum given by Eq. (27).

Two discharges can be said to be similar if for equal applied voltages they carry equal currents, irrespective of their dimensions. The similarity principle states that when a given discharge $\mathrm{A}$ is operated under particular condition, a discharge B can be operated with the gas density of discharge A multiplied by a factor $a$ and the linear dimensions multiplied by a factor $1 / a$, then the space charge density and the current density scales by a factor $a^{2}$. So for similar discharges the requirement of equal currents leads to $\alpha_{\mathrm{A}} L_{\mathrm{A}}=\alpha_{\mathrm{B}} L_{\mathrm{B}}$, or $\alpha_{\mathrm{B}}=\alpha_{\mathrm{A}} / a$. Also, since $V_{\mathrm{D}}$ is the same for both discharges, $E_{\mathrm{B}}=$ $E_{\mathrm{A}} / a$. Then the pressure scales as $p_{\mathrm{B}}=p_{\mathrm{A}} / a$ and we have $\alpha_{\mathrm{B}} / p_{\mathrm{B}}=\left(\alpha_{\mathrm{A}} / a\right) /\left(p_{\mathrm{A}} / a\right)=\alpha_{\mathrm{A}} / p_{\mathrm{A}}$ so $\alpha / p$ is invariant. Similarly for similar discharges $p L_{\mathrm{c}}$ is invariant, and $E / p$ or $E / n_{\mathrm{g}}$ is invariant. The ratio $E / p$, is a measure of the energy gain of a charged particle between collisions, and is referred to as the reduced electric field. Typically, it was found that the breakdown conditions and the current growth depend only on scaling parameters $E / n_{\mathrm{g}}$, and $p L$ (Phelps, 2001), such as in case of the Paschen curve. Additionally, the discharge voltage can be expressed as a function of the reduced current density $J / p^{2}$, and Škoro et al. (2008) have shown that the $J / p^{2}$ scaling law can be used to recognise the transition between different plasma regimes, from Townsend discharge, to the normal glow, and further to the abnormal glow, similar to the $V_{\mathrm{D}}-I_{\mathrm{D}}$ characteristic presented in Figure 1.

\section{B. Secondary electrons}

The emission of secondary electrons as a result of ion or neutral bombardment of a metallic surface plays an important role in the discharge physics. Typical energy of emitted electrons leaving the cathode surface is about few eV. Since the cathodes are held at negative potentials, the secondary electrons are accelerated away from the cathode surface with an energy equal to the potential drop ion the cathode sheath. These electrons sustain the discharge by ionization of the neutral gas, and generation of ions which then bombard the cathode releasing more secondary electrons. Ion- and neutral-induced secondary electron emission has been studied both theoretically and experimentally for decades (Abroyan et al., 1967; Hasselkamp, 1992; Parilis and Kishinevskii, 1960).

The secondary electron emission yield or coefficient $\gamma_{\text {see }}$ is defined as the number of secondary electrons emitted per incident ion or neutral. For low energies of impinging ion, the secondary electron emission yield is independent of the velocity of the bombarding particle, since the electron emission occurs due to transfer of the incoming ion or atom's potential energy to an electron in the cathode surface. This constant secondary electron emission yield is attributed to an Auger process referred to as potential emission. For impinging ion or neutral energies above several hundreds $\mathrm{eV}$ to $\mathrm{keV}$ the energy dependent portion of the yields is called kinetic emission. Kinetic emission, occurs when a bombarding particle transfers sufficient kinetic energy to an electron in the cathode, starts contributing to the total yield at a threshold energy of around a few hundred electron volts and dominates at higher energies. Both experimental data and theory predict a linear dependence of the secondary electron emission yield on the bombarding energy close to the threshold energy, and linear dependence on the bombarding velocity at higher energies (Abroyan et al., 1967; Baragiola et al., 1979; Cawthron, 1971; Hasselkamp, 1992; Parilis and Kishinevskii, 1960). At much higher energies, experimental data shows that the electron yield starts decreasing with increasing bombarding velocity. This occurs for a bombarding energy of around 
$100 \mathrm{keV}$ for $\mathrm{H}^{+}$(Hasselkamp, 1992).

The two different mechanisms are considered to be coexisting, so the total electron emission yield is written as

$$
\gamma_{\text {see }}=\gamma_{\mathrm{p}}+\gamma_{\mathrm{k}}
$$

where $\gamma_{\mathrm{p}}$ and $\gamma_{\mathrm{k}}$ are the contributions from potential and kinetic emission to the total yield, respectively. In addition to the energy of the impacting particle the secondary electron emission yield depends on the cathode material, but rarely exceeds 0.2 for an ion energy below $1 \mathrm{keV}$. The condition of the cathode significantly affects the ion induced secondary electron emission yield. Clean metals, i.e. metals free of oxidation, gas adsorption, and other contamination, generally have a lower kinetic emission yield than contaminated metals (Phelps and Petrović, 1999). Phelps et al. (Phelps and Petrović, 1999; Phelps et al., 1999) give the secondary electron emission yield in the energy range $10-10000 \mathrm{eV}$ for both clean and dirty metal surfaces bombarded by $\mathrm{Ar}^{+}$-ions and Ar neutrals.

\section{The cathode sheath}

All plasmas are separated from the surrounding walls by a sheath. The sheath properties determine the energy and flux of charged particles that bombard the wall surface. We have seen in section III, and in particular in figure 5 (a), that most of the potential drop over a dc glow discharge is across the cathode sheath. The relation between the current density, the voltage drop across the sheath and the sheath thickness was derived by Child (1911), assuming that the initial ion energy is negligible compared to the sheath potential (see also Lieberman and Lichtenberg (2005, Section 6.2.)), giving

$$
J=\frac{4}{9} \epsilon_{0}\left(\frac{2 e}{M_{\mathrm{i}}}\right)^{1 / 2} \frac{V_{\mathrm{c}}^{3 / 2}}{d_{\mathrm{c}}^{2}}
$$

which is called the Child law or the collisionless ChildLangmuir law. The Child law is valid when the sheath potential is large compared to the average energy of the electrons. Similar realation was derived by Langmuir (1913) for electrons emitted from a hot cathode approaching a cold anode.

In the collisional regime where the pressure is high enough that the charged species interact frequently with neutral gas species we can assume that the ion-neutral mean free path $\lambda_{\mathrm{i}}$ is independent of the ion velocity (Lieberman and Lichtenberg, 2005, Section 6.6.). This gives the collisional Child law

$$
J=\left(\frac{2}{3}\right)\left(\frac{5}{3}\right)^{3 / 2} \epsilon_{0}\left(\frac{2 e \lambda_{\mathrm{i}}}{\pi M_{\mathrm{i}}}\right)^{1 / 2} \frac{V_{\mathrm{c}}^{3 / 2}}{d_{\mathrm{c}}^{5 / 2}}
$$

Alternatively, assuming that diffusion of ions is negligible compared to drift due to electric field and take the ion mobility $\mu_{\mathrm{i}}$ to be independent of the ion velocity to get

$$
J=\frac{9}{8} \epsilon_{0} \mu_{\mathrm{i}} \frac{V_{\mathrm{c}}^{2}}{d_{\mathrm{c}}^{3}}
$$

which is referred to as the Mott-Gurney law. It was derived to describe the current at the interface of a semiconductor and insulator (Mott and Gurney, 1948, Chapter V) and later adapted to describe the current through the discharge sheath by Cobine (1958). Equation (33) is valid only at very high pressures (low drift velocities). We note here that the scalings of the current density with both $V_{\mathrm{c}}$ and $d_{\mathrm{c}}$ in Eq. (32) are different from Eq. (33). Further discussion of the collisionless Child-Langmuir law and the collisional Mott-Gurney law is given by Benilov (2009). It has been demonstrated by experiments that the MottGurney law (Eq. (33)) applies to a dc glow discharge in hydrogen (Lisovskiy et al., 2016) and nitrogen (Lisovskiy et al., 2014) for most of the pressure range from 10 to $333 \mathrm{~Pa}$.

\section{THE DC GLOW DISCHARGE SPUTTERING SOURCE}

An important process that can occur at the cathode in the glow discharge is sputtering, which is ejection of the atoms from the cathode surface by the impinging ions. Sputtering can occur if the voltage applied to the cathode is sufficiently high. When the ions and fast neutrals from the plasma bombard the cathode target, they release secondary electrons and they also release atoms of the cathode material.

\section{A. Sputter yield}

Sputtering is the ejection of atoms due to bombardment of a solid or a liquid surface (the target) by energetic particles, often ions (Behrisch and Eckstein, 2007). When the cathode surface is struck by an impinging particle in a given energy range some atoms in the surface, referred to as primary knock on atoms, may gain substantial amount of the energy of the incoming ion through the collision. They in turn sputter or strike other atoms in the surface transferring momentum yet again (Behrisch and Wittmaack, 1991). The sputter yield also depends on the ion incident angle. The sputter yield increases with increasing angle of incidence and maximum occurs in the range between $60^{\circ}$ and $80^{\circ}$ (Oechsner, 1975).

The sputter yield $Y$ is defined as the mean number of atoms removed from the target surface for each incident ion. The maximum transferable energy in a collision has to be larger than the surface binding energy or $\mathcal{E}_{\text {th }}+\mathcal{E}_{\mathrm{sp}}>$ $\mathcal{E}_{\mathrm{sb}} / \Lambda$ where $\mathcal{E}_{\mathrm{sb}}$ is the surface binding energy (heat of sublimation) of the target material, $\mathcal{E}_{\mathrm{sp}}$ is the binding energy of a projectile to the target surface $\left(\mathcal{E}_{\mathrm{sp}}=0\right.$ for noble gas ions) and $\Lambda=4 M_{\mathrm{i}} M_{\mathrm{t}} /\left(M_{\mathrm{i}}+M_{\mathrm{t}}\right)^{2}$ is the energy transfer factor in a binary collision and $M_{\mathrm{i}}$ and $M_{\mathrm{t}}$ are the masses of the projectile and the target atom, respectively 
(Eckstein, 2007). The minimum ion energy required for sputtering to take place is known as the threshold energy for sputtering and is given by (Yamamura and Tawara, 1996)

$$
\mathcal{E}_{\mathrm{th}}=\left\{\begin{array}{ll}
\mathcal{E}_{\mathrm{sb}}\left(1+5.7 \frac{M_{\mathrm{i}}}{M_{\mathrm{t}}}\right) / \Lambda & \text { if } \quad M_{\mathrm{i}} \leq M_{\mathrm{t}} \\
\mathcal{E}_{\mathrm{sb}} \times 6.7 / \Lambda & \text { if } \quad M_{\mathrm{i}} \geq M_{\mathrm{t}}
\end{array} .\right.
$$

Yamamura and Tawara (1996) give the various empirical formulas for the sputter yield as a function of ion bombarding energy and data for various combinations of ions and target materials. In the energy range of interest here, $20-5000 \mathrm{eV}$, the sputter yield increases with increasing incident ion energy. In this energy range the sputter yield can be approximated by

$$
Y\left(\mathcal{E}_{\mathrm{i}}\right)=a \mathcal{E}_{\mathrm{i}}^{b}
$$

where $a$, a material dependent parameter, and $b \sim 0.5$ are fit parameters that are given for a particular combination of bombarding ion and target materials (Anders, 2017). As an example for $\mathrm{Ar}^{+}$bombarding a $\mathrm{Cu}$ target $a=$ 0.1421 and $b=0.468$, while for the self-sputtering $\mathrm{Cu}^{+}$ bombarding a $\mathrm{Cu}$ target $a=0.0691$ and $b=0.556$.

Computer codes such as TRIM (Transport of Ions in Matter) (Biersack and Haggmark, 1980), SRIM (Stopping and Range of Ions in Matter) (Ziegler et al., 2008, 2010) and TRIDYN (A TRIM simulation code including dynamic composition changes) (Möller and Eckstein, 1984; Möller et al., 1988) are used to calculate the sputter yield for a given inpacting species as a function of the energy of the incident particle. They use a binary collision model and follow the incident particles and all of its cascade atoms until they sputter or their energy is too low to escape the surface potential.

\section{B. Energy distribution of sputtered atoms}

The atoms sputtered off the cathode target are considerably more energetic than thermally evaporated atoms (a few eV as compared to about a tenth of an eV). Usually it is desirable to maintain this initial kinetic energy of the sputtered atoms, since it has favourable effects on the film growth (Petrov et al., 1993). Relatively low pressures are normally desired to minimize scattering of the sputtered atoms. The sputtering process is, therefore, normally a line-of-sight process where the deposition flux cannot be easily controlled, since it consists of neutral atoms. This broad distribution has been measured for sputtered neutrals (Stuart et al., 1969) and is predicted by the Thompson random collision cascade model (Thompson, 1968, 1981). According to the Sigmund-Thompson theory the energy distribution function can be approximated by

$$
f_{\mathrm{S}-\mathrm{T}} \propto \frac{\mathcal{E}}{\left(\mathcal{E}+\mathcal{E}_{\mathrm{sb}}\right)^{3-2 m}}
$$

where $\mathcal{E}_{\mathrm{sb}}$ is the binding energy of the target material and $m$ is the exponent in the interaction potential applied $V(r) \propto r^{-m}$ (Hofer, 1991). Often $m$ is taken to be 0.2 . This model predicts an energy spectrum that peaks sharply at $\frac{1}{2} \mathcal{E}_{\mathrm{sb}}$, followed by a gradual decrease to higher energies $\left(\propto 1 / \mathcal{E}^{2}\right)$. The energy distribution of atoms ejected from a target is expected to be independent of the nature of the incident ion as well as the crystal structure of the target. The angular distribution of the sputtered atoms is often described as a cosine distribution. That says that the relative amount of material sputtered at any particular angle can be compared to the amount sputtered at normal incidence times the cosine of the angle from normal incidence. This means that the overall distribution can be drawn as a ellipse, and in three dimensions, the distribution would appear as an ellipsoid centered on the ion impact point. For a more detailed discussion of the ion energy and ion angular distribution of the sputtered material the reader should consult the reviews given by Hofer (1991) and Gnaser (2007) or the original work of Thompson $(1968,1981)$ and Sigmund (1969).

If the sputtered material is subsequently ionized the ion energy distribution generally shows a narrow low energy peak, due to thermalized atoms which are accelerated by the difference between plasma potential (several $\mathrm{eV}$ ) and grounded electrode, and a broad distribution at higher energies which originates from the sputtered neutrals which have been ionized by electron impact within the plasma (see e.g. Andersson et al. (2006)). Due to the small mass of the electron the electron impact ionization does not change the energy of the resulting ion by much.

\section{Diode sputtering}

Sputtering in gas discharges was discovered in the mid 19th century (Grove, 1852), while film formation utilizing sputter deposition was first reported by Wright (1877a,b) a few decades later. Sputtering of thin films had already found commercial application by the 1930s (Fruth, 1932) but gained significant interest in the late 1950s and early 1960 s with improved vacuum technology and the realization that a wide range of materials could be deposited using sputtering (Kay, 1962; Thornton and Greene, 1994; Westwood, 1976) including dielectrics, but then by applying rf voltage (Anderson et al., 1962). There have been a number of review articles and books covering de glow discharge sputter deposition (Chopra, 1969; Kay, 1962; Vossen and Cuomo, 1978; Westwood, 1976).

For decades the dc glow discharge was used as a sputter source, commonly referred to as diode sputtering. A schematic of the diode sputter arrangement is shown in Figure 8. The distance between cathode and anode is generally short, so typically only a short anode zone is present together with the cathode dark space and the negative glow, where the slightly positive plasma potential returns back to zero at the anode. They are often 


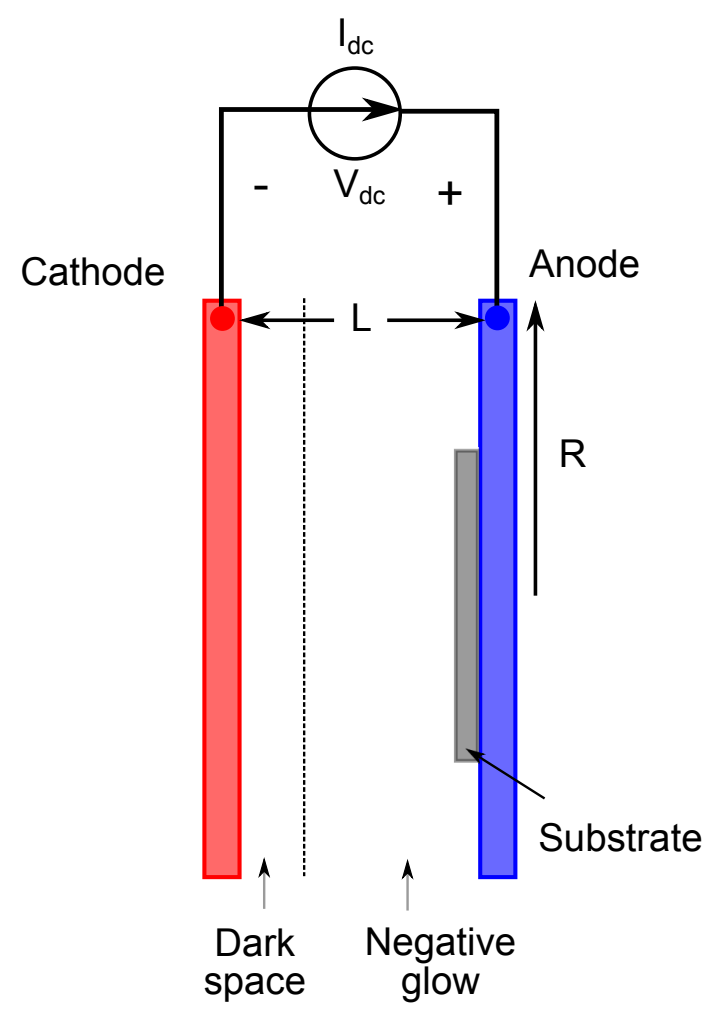

FIG. 8 Low aspect ratio dc glow discharge used for sputtering or the dc diode sputter source. The cathode is the material source. The interelectrode separation is a few times that of the cathode dark space and the negative glow extends almost to the anode. The substrate is placed on the anode.

low aspect ratio, the interelectrode separation is small compared to the size of the electrode, $(L / R<1$ for a cylindrical configuration) and mainly used for sputtering of metals. The cathode serves as a target for ion impact sputtering and is the material source. Cathode diameters are typically in the range $10-30 \mathrm{~cm}$ while the spacing between the cathode and anode is 5 to $10 \mathrm{~cm}$. The interelectrode separation is a few times that of the cathode dark space. This configuration is referred to as an obstructed dc glow discharge. If the length of a glow discharge is less than $L_{\mathrm{c}}$ at the Paschen minimum value given by Eq. (29), then the voltage drop over the cathode fall rises above the Paschen minimum value, $V_{\mathrm{c}, \mathrm{min}}$. Almost all the voltage appears across cathode sheath (dark space, i.e. cathode fall). The negative glow extends almost to the anode and the positive column is commonly absent in these short discharges. Typical dc glow discharge for sputter deposition requires a negatively charged cathode at $2000-$ $5000 \mathrm{~V}$ and a grounded surface anode. The substrate, on which the sputtered atoms are deposited, is placed on the other electrode, which is the anode. The substrate holder may be grounded, floating, biased, heated, cooled, or some combination of these.

In the dc sputtering diode configuration, the ions that impinge on the target surface do not have the full cath- ode fall potential. This is due to the collisional sheath (Eq.(13) and Eq.(14)) where the working gas pressure is high enough to allow charge-exchange collisions and momentum transfer collisions (thermalization) between the accelerating ions and the working gas neutrals. The consequence is that there is a broad energy spectrum of ions and high energy neutrals that impinge on the target surface. The higher the gas pressure, the lower the mean energy of particles that bombard the target. Since the discharge is maintained by secondary electron emission the operating pressure must be high enough so that the secondary electrons are not lost to the anode or to the grounded surfaces before performing ionization. These pressures are higher than preferred for optimum transport of the sputtered deposition atoms due to scattering by the gas atoms. Hence, there is a narrow pressure range around $2-4 \mathrm{~Pa}$ for dc glow discharge sputtering to be viable. At this pressure the cathode dark space extends about $1-2 \mathrm{~cm}$ from the cathode. The discharge maximum current density is roughly $1 \mathrm{~mA} / \mathrm{cm}^{2}$ and the deposition rate ten $\mathrm{nm} / \mathrm{min}$ at best. The dc sputter source is generally weekly ionized with ionization fraction of the order of $10^{-4}$. Furthermore, a conventional dc diode discharge is not operable if the cathode surface is insulating. This would be the case if the cathode was itself an insulator, or if the cathode was under operation in reactive gas such as oxygen which might make the surface of the cathode insulating. This results in either case in extremely low discharge currents and very low sputtering rate.

The disadvantages of dc diode sputter deposition include low sputtering rate, target poisoning by reactive contaminants, surface heating due to electrons accelerated away from the target, and that only electrical conductors can be used as sputtering targets. Also the sputtering power efficiency (sputtered atoms/ion-volt) is relatively low in these discharges as they operate at high voltage and this efficiency decreases with increasing energy.

\section{THE MAGNETRON SPUTTERING DISCHARGE}

The high discharge voltage and high operating pressure of dc discharge sputtering source are limiting to its application. In order to lower the discharge voltage and expand the operation pressure range, the lifetime of the electrons in the target vicinity had to increase in order to enhance the ionisation. This was achieved by applying a static magnetic field applied to confine the secondary electrons in the vicinity of the cathode target (Gill and Kay, 1965; Wasa and Hayakawa, 1969). This is referred to as a magnetron sputtering discharge. With the introduction of magnetron sputtering the disadvantages of diode sputtering, such as poor deposition rate, were overcome as the operating pressure could be reduced, while maintaining the energy of the sputtered species, often resulting in improved film properties. A schematic of a dc planar magnetron sputtering configuration is shown 
in Figure 9. In the planar configuration the magnetron sputtering discharge is simply a diode sputtering arrangement with the addition of magnets directly behind the cathode. The magnetic field lines go out in the centre of the cathode and go back into the cathode at the annular (Chapin, 1974; Waits, 1978). The magnetic field is arranged so that it appears parallel to the cathode surface. In a conventional dc magnetron sputtering (dcMS) discharge the cathode is kept at a constant negative voltage. Sometimes a planar magnetron discharge consists of a planar cathode (sputtering source or target) parallel to an anode surface. However, in most cases the anode is the grounded shield around the magnetron target (as seen in Figure 9).

A simple model that gives an estimate of the minimum voltage needed to sustain a magnetron sputtering discharge was set forth by Thornton (1978). The number of electron-ion pairs created by each secondary electron that is trapped in the target vicinity is given as

$$
\mathcal{N} \approx \frac{V_{\mathrm{d}}}{\mathcal{E}_{\mathrm{c}}}
$$

where $\mathcal{E}_{\mathrm{c}}$ is the energy loss per electron-ion pair created with the flow of secondary electrons into the plasma as the source of energy (Depla et al., 2010; Lieberman and Lichtenberg, 2005; Thornton, 1978; Thornton and Penfold, 1978). For argon and high energy electrons $\mathcal{E}_{\mathrm{c}} \approx 20$ $\mathrm{V}$. However, not all the secondary electrons are confined in the target vicinity. To account for the electrons that are not trapped we define an effective secondary electron emission coefficient

$$
\gamma_{\mathrm{see}, \mathrm{eff}}=m \epsilon_{\mathrm{e}}(1-r) \gamma_{\mathrm{see}}
$$

where $\epsilon_{\mathrm{e}}$ is the fraction of the electron energy that is used for ionization before being lost, $m$ is a factor that accounts for secondary electrons ionizing in the sheath and $r$ is the recapture probability of secondary electrons. To sustain the discharge the condition

$$
\gamma_{\text {see, eff } \mathcal{N}}=1
$$

has to be fulfilled. This defines the minimum voltage to sustain the discharge as

$$
V_{\mathrm{D}, \min }=\frac{\mathcal{E}_{\mathrm{c}}}{\beta \gamma_{\mathrm{see}, \mathrm{eff}}}
$$

$\beta$ is the fraction of ions that return to the cathode. Equation (39) is often referred to as the Thornton equation. The basic assumption is that acceleration across the sheath is the main source of energy for the electrons (Thornton, 1978). Above breakdown, the parameters $m$, $\beta, \epsilon_{\mathrm{e}}$ and $r$ can vary with the applied voltage, so we can rewrite the Thornton equation for any voltage as (Depla et al., 2009)

$$
\frac{1}{V_{\mathrm{D}}}=\frac{\beta m \epsilon_{\mathrm{e}}(1-r)}{\mathcal{E}_{\mathrm{c}}} \gamma_{\mathrm{see}}
$$

Thus a plot of the inverse discharge voltage $1 / V_{\mathrm{D}}$ against $\gamma_{\text {see }}$ should then give a straight line through the origin. However, there are indications that the magnetron sputtering discharge is not fully maintained by acceleration of secondary electrons emitted from the cathode target. When experimentally determined $1 / V_{\mathrm{D}}$ is plotted against $\gamma_{\text {see }}$ it does not go through the origin (Depla et al., 2009). This suggests that Ohmic heating within the dense plasma, that hovers next to the cathode surface, seems to play a significant role as well (Brenning et al., 2016).

The main advantage of the planar magnetron is that the sputtered material is transported in the direction normal to the cathode plane and will uniformly coat a surface that is translated past the magnetron target. Conventional planar dcMS sources are commonly operated using argon as the working gas in the pressure range 0.1 - 1.5 $\mathrm{Pa}$ and the applied cathode voltage in the range of $300-700 \mathrm{~V}$. This leads to current densities of the order of $4-60 \mathrm{~mA} / \mathrm{cm}^{2}$ and power densities of several tens of $\mathrm{W} / \mathrm{cm}^{2}$ (Waits, 1978). Magnetron sputtering configurations use a magnetic field $(\sim 20-50 \mathrm{mT})$, usually from permanent magnets near the target (cathode) surface, to confine the electrons near the surface. The electron density in the substrate vicinity is typically in the range $10^{15}-10^{17} \mathrm{~m}^{-3}$. The static deposition rate is in the range $20-200 \mathrm{~nm} / \mathrm{min}$. The degree of ionization of the sputtered material is generally very low, often on the order of $1 \%$ or less. The majority of the ions bombarding the substrate are ions of the working gas as the mean free path for the sputtered material with respect to electron impact ionization is over $50 \mathrm{~cm}$.

It is referred to as a balanced planar magnetron, when an axisymmetric magnetic field is applied with a permanent magnet behind the cathode, in such a way that the magnetic field lines start and return at the magnet. To increase the ion flux to the substrate the magnetic field lines can be configured such that they do not all close at the cathode surface. This is referred to as unbalanced magnetron sources (Window and Savvides, 1986). Then some of the electrons are lead towards the substrate, and by ambipolar diffusion, ions as well. Hence, by altering the magnetic field configuration, thin film growth can be achieved with varying ion and electron flux.

In the rotatable cylindrical magnetron sputtering discharge the magnet assembly is installed inside the cylindrical cathode target (Wright and Beardow, 1986). The target can rotate during the sputtering so it erodes uniformly and the target utilization is as high as $90 \%$. This configuration also allows for deposition on large area substrates. In the cylindrical configuration the target surface area may be in hundreds and up to tens of thousands of square $\mathrm{cm}$. These rotatable targets are essential 


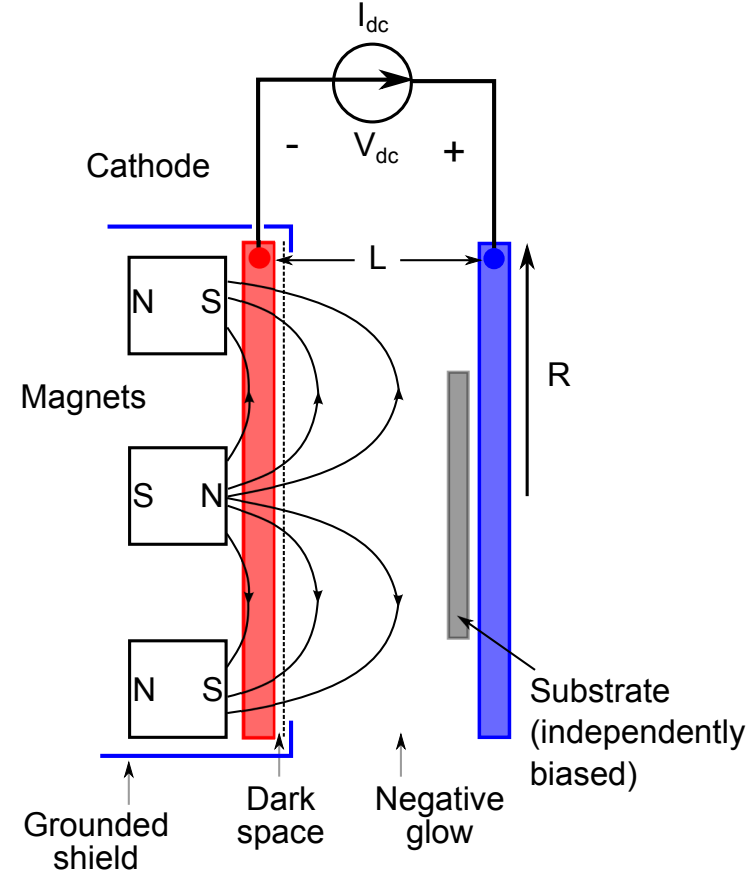

FIG. 9 A schematic of the dc planar magnetron discharge used for sputtering. Permanent magnets behind the cathode target confine the secondary electrons in the vicinity of the target surface. The anode is often a grounded shield around the magnetron target.

for deposition on large area glass for architectural and automotive applications as well as for the production of flat panel displays and photovoltaic solar cells (Blondeel et al., 2009).

\section{A. Pulsed magnetron sputtering discharges}

The asymmetric bipolar dc sputtering discharge was developed to optimize the deposition of insulating films from conductive targets with reactive sputtering (Schiller et al., 1993; Sellers, 1998). Pulsing the magnetron discharge in the medium frequency range $(10-250 \mathrm{kHz})$ when depositing insulating films can significantly reduce the formation of arcs and, consequently, reduce the number of defects in the resulting film. In asymmetric bipolar mode the target is pulsed between the normal operating voltage and a slightly positive (roughly $10-20 \%$ of the negative voltage amplitude) voltage for a short duration.

More recently high-power pulsed magnetrons in unipolar mode have been proposed for highly ionized sputtering (Gudmundsson et al., 2012). High power impulse magnetron sputtering (HiPIMS) is a sputtering technique where a high density plasma is created by applying high power pulses at low frequency and low duty cycle to a magnetron sputtering device. Then the peak electron density is in the range of $10^{18}-10^{19} \mathrm{~m}^{-3}$ which corresponds to an electron impact ionization mean free path for the sputtered material of the order of $1 \mathrm{~cm}$ or less. Thus the sputtered material is highly ionized. For the ionized material the ion energy as well as the ion flux can easily be controlled over a wide range making it possible to tailor phases, microstructure, defect density, and composition of growing film as well as utilizing the metal ions for etching (Helmersson et al., 2006). Furthermore, as the sputtered material is ionized it enables guiding and aligning of the deposition material.

\section{CHEMICAL ANALYSIS WITH GLOW DISCHARGES}

Low pressure glow discharges constitute a standard method for chemical analysis of metals and metallurgical samples. This method is referred to as glow discharge optical emission spectrometry (GD-OES) and is currently a well established technique in particular for surface and interface characterization (Broekaert, 2003; Nelis and Payling, 2003). The method was suggested by Grimm in the late sixties (Grimm, 1968) for bulk analysis of solid conducting samples. Later it was developed into allowing for quantitative depth profiling of surfaces and coatings (Pons-Corbeau, 1985). By applying radio frequency the analysis of insulating materials was made possible (Duckworth and Marcus, 1989). The sample to be analyzed is mounted on an o-ring on the outside of the plasma source, so that sample placement is rather easy and simple. Argon gas is introduced into the chamber of roughly $200-500 \mathrm{~Pa}$. As voltage is applied to the sample, which is the cathode, a glow discharge is created and sputtering is initiated. The plasma forms in a hollow anode glow discharge. The glow discharge is connected to an optical spectrometer. The recorded intensity of an atomic emission line is a measure of the number of atoms of a particular element in the plasma and thus the concentration of the element in the sample analyzed. Glow discharge atomic emission spectra have narrow lines as a result of the low Doppler and pressure broadening. Analytical glow discharges are typically operated as abnormal glow discharges.

Similarly, mass spectrometry can also be applied for elemental analysis of solids, and then the process is referred to as glow discharge mass spectrometry (GDMS) (Coburn and Harrison, 1981; Harrison et al., 2003). Then a mass spectrometer is attached to the discharge and the ions of the material sputtered off the sample are sampled through an orifice. One advantage of elemental mass spectrometry is that is permits easier quantitative interpretation over the line rich optical emission spectra. They also have the ability to obtain the full periodic table, including information on isotopes with detection limit that is $2-3$ orders of magnitude better than OES. Also, another advantage is achieved by using a pulsed glow discharge that gives a temporal separation of discharge gas ions and the analyte ions, particularly with the application of a time-of-flight mass spectrometer. 


\section{THE PENNING DISCHARGE}

In the Penning discharge a strong axial magnetic field is applied to the dc discharge (Hooper, 1969). They can operate at very low pressures since the electrons have a very long ionization mean free paths due to being magnetically confined. Penning discharges can provide energetic ions of any material that can be gasified. The early application of this arrangement of the electric and magnetic field was when Penning introduced a manometer for low gas pressures, for the pressure range of $0.01 \mathrm{mPa}-$ $0.01 \mathrm{~Pa}$, consisting of a glow discharge tube in a magnetic field (Penning, 1937). Its operation is based on the fact that the current through the discharge is proportional to the pressure. This manometer is very simple in use and gives an instantaneous indication of pressure. This concept is also utilized in a combination of homogeneous magnetic field and electrostatic quadrapole potential arranged in such a way that it can trap a single particle (Brown and Gabrielse, 1986). This is know as a Penning trap. Electrons are initially introduced into the trap via field emission. This creates an electron beam that ionizes residual gas to produce slow electrons which are then captured in the trap. Thus it allows for measuring physical quantities of individual particles at very high precision.

\section{HOLLOW CATHODE DISCHARGES}

Hollow cathode discharges are gas discharges between a hollow structured cathode and an arbitrarily shaped anode. The hollow cathode cavity can present planeparallel, cylindrical, or spherical geometry. The open side of the cathode faces the anode side of the discharge. The anode can be remote. The hollow cathode discharge is based on an entrapment of electrons inside the hollow cathode. The cathode walls are made of conducting, refractory materials kept at the cathode potential. When energetic electrons emitted from one cathode wall are accelerated across the sheath towards the opposite wall they reach an identical sheath on the opposite side with the same but opposite electric field and they are reflected back. Thus the electrons are trapped and forced to oscillate between the opposite sheaths. During these oscillations electrons will undergo inelastic collisions with gas atoms and therefore increase the probability of ionization and leading to a very dense plasma inside the cathode volume. This mechanism is referred to as the hollow cathode effect (Kolobov and Tsendin, 1995). The plasma existing at the inter-electrode space can penetrate the hollow cathode, thus assuring a strong interaction between the plasma and the cathode internal surface. The plasma created within the hollow cathode is then finally forced out of the cathode along with the flowing gas. For further information on hollow cathode discharges the reader is referred to the reviews by Mavrodineanu (1984), Pillow (1981), Delcroix and Trindade (1974), and Muhl and Pérez (2015).
Hollow cathodes have been utilized in different discharge devices, working under varying conditions in widely extended ranges. Hollow cathode discharges are widely used as electron and ion sources and as a source of intense line radiation. Initially they were used as thermionic emitters to produce electron beams. The large area of the hollow cathode emitter lead to extended lifetime of the emitter surface. These were operated in high vacuum. But hollow cathodes are also operated as plasma discharges. The primary advantage of the hollow cathode discharge is that a high degree of ionization can be sustained, in the negative glow, even for a rare gas/metal vapor mixture.

Hollow cathodes are critical components of Hall thrusters where they provide the electrons that ionize the neutral propellant gas to create the plasma which is then directed to create thrust and supply the electrons that neutralize the exhaust ion beam in gridded ion thrusters (Goebel and Katz, 2008, Chapter 6). The currents drawn may be rather significant or up to $100 \mathrm{~A}$. The hollow cathode discharges have also been used as sputter sources for sputter deposition (Muhl and Pérez, 2015). The hollow cathode magnetron sputtering discharge is an example where a hollow cathode is combined with a dc magnetron sputtering discharge in order to achieve high ionization fraction of the deposition material (Klawuhn et al., 2000).

\section{A. Plasma display panels}

Another common use of dc plasmas is in plasma display panels (PDP). The PDP is based on small gas discharges: micro-discharges, to generate a basic color (red, green or blue) of a pixel. There are two predominant ways of limiting the current in PDPs; dc display current limiting, which is based on current-limiting properties of the system resistance, and the ac display current limiting, which is based on the capacitance generated by a dielectric glass layer of the plasma cell. More detailed discussion on PDPs can found in the review articles given by Boeuf (2003) and Weber (1985).

The more commonly used ac PDP consists of two glass plates placed at a distance of $100-200 \mu \mathrm{m}$ from each other. The region between the plates is filled with a gas at a pressure of roughly $60 \mathrm{kPa}$. The PDP discharge operates in glow discharge regime since the $p L$ value of a such system is in range of several $\mathrm{kPa} \mathrm{cm}$. The lines of electrodes are placed on the outer sides of both glass plates, in such a way that the electrodes of one plate are placed perpendicular to the electrodes of the other plate in order to form the rows and columns of a display. At each intersection between a row and a column electrode, a discharge can be formed, independent of the other intersections, by applying suitable voltage pulses to the electrodes.

Each pixel consists of three plasma cells corresponding to three basic colors (red, green and blue). On one side the plasma cell is covered with a thin layer of phos- 
phor (red, green, or blue) and on the other side with a thin layer of another material, typically magnesium oxide $(\mathrm{MgO})$. The discharge gas is typically a mixture of rare gases (xenon/neon/helium) which emit UV photons. The UV radiation activates color phosphors and visible light is emitted from each pixel. The $\mathrm{MgO}$ layer is very important for efficiency and longevity of the cell; it has a large secondary electron yield (keeping the operating voltage relatively low), high transmission in the visible range, and good resistance to sputtering (long lifetime).

\section{SUMMARY}

We have given an overview of the dc discharge and in particular the dc glow discharge. The dc discharge typically has a negative cathode at one end and a positive anode at the other end, separated by a gas filled gap, enclosed in a long glass cylinder. We discussed the current-voltage characteristics of the discharge as well as the distinct structure that develops in the glow discharge region, for discharge current in the range from $\mu \mathrm{A}$ to $\mathrm{mA}$ at operating pressure of $0.5-300 \mathrm{~Pa}$. The dc glow discharge exhibits a distinct structure and is maintained by the emission of secondary electron emission from the cathode target. The dc glow discharge has been used as a sputter source, often operated as an obstructed abnormal glow discharge and the required applied voltage is in the range $2-5 \mathrm{kV}$. The magnetron sputtering discharge was developed in order to lower the discharge voltage and expand the operation pressure. We also briefly discussed other configurations of the dc discharge including its use in chemical analysis of surfaces, the Penning discharge and the hollow cathode discharges.

\section{Acknowledgments}

This work was partially supported by the Icelandic Research Fund Grant No. 163086, the Swedish Government Agency for Innovation Systems (VINNOVA) contract no. 2014-04876, and by the DFG (German Science Foundation) within the framework of the Coordinated Research Center SFB-TR 87. We are thankful to Dr. P. J. Petty at LPGP Université Paris Sud for drawing Figure 2.

\section{References}

Abroyan, I. A., M. A. Eremeev, and N. N. Petrov (1967). Excitation of electrons in solids by relatively slow atomic particles. Physics-Uspekhi 10 (3), 332-367.

Alves, L. L. (2007). Fluid modelling of the positive column of direct-current glow discharges. Plasma Sources Science and Technology 16(3), 557-569.

Anders, A. (2017). Tutorial: Reactive high power impulse magnetron sputtering (R-HiPIMS). Journal of Applied Physics 121(17), 171101.
Anderson, G. S., W. N. Mayer, and G. K. Wehner (1962). Sputtering of dielectrics by high-frequency fields. Journal of Applied Physics 33(10), 2991-2992.

Andersson, J. M., E. Wallin, E. P. Münger, and U. Helmersson (2006). Energy distributions of positive and negative ions during magnetron sputtering of an $\mathrm{Al}$ target in $\mathrm{Ar} / \mathrm{O}_{2}$ mixtures. Journal of Applied Physics 100(3), 033305.

Baragiola, R. A., E. V. Alonso, J. Ferron, and A. Oliva-Florio (1979). Ion-induced electron emission from clean metals original research article. Surface Science 90(2), 240-255.

Behrisch, R. and W. Eckstein (2007). Introduction and overview. In R. Behrisch and W. Eckstein (Eds.), Sputtering by Particle Bombardment: Experiments and Computer Calculations from Threshold to MeV Energies, Volume 110 of Topics in Applied Physics, Chapter 1, pp. 1-10. Berlin Heidelberg: Springer Verlag.

Behrisch, R. and K. Wittmaack (1991). Introduction. In R. Behrisch and K. Wittmaack (Eds.), Sputtering by Particle Bombardment III, Volume 64 of Topics in Applied Physics, pp. 1-13. Berlin Heidelberg: Springer Verlag.

Benilov, M. S. (2009). The Child-Langmuir law and analytical theory of collisionless to collision-dominated sheaths. Plasma Sources Science and Technology 18(1), 014005.

Bernstein, I. B. and T. Holstein (1954). Electron energy distributions in stationary discharges. Physical Review 94 (6), 1475-1482.

Biersack, J. P. and L. G. Haggmark (1980). A Monte Carlo computer program for the transport of energetic ions in amorphous targets. Nuclear Instruments and Methods 174 (1-2), 257-269.

Blondeel, A., P. Persoone, and W. De Bosscher (2009). Rotatable magnetron sputter technology for large area glass and web coating. Vakuum in Forschung und Praxis 21(3), 6-13.

Boeuf, J. P. (2003). Plasma display panels: physics, recent developments and key issues. Journal of Physics D: Applied Physics 36(6), R53-R79.

Booth, J. P., M. Fadlallah, J. Derouard, and N. Sadeghi (1994). Electric field measurements in discharges by $2+1$ photon laser Stark spectroscopy of atomic hydrogen. $A p$ plied Physics Letters 65(7), 819-821.

Brenning, N., J. T. Gudmundsson, D. Lundin, T. Minea, M. A. Raadu, and U. Helmersson (2016). The role of Ohmic heating in dc magnetron sputtering. Plasma Sources Science and Technology 25(6), 065024.

Broekaert, J. A. C. (2003). Optical emission spectrometry with glow discharges. In R. K. Marcus and J. A. C. Broekaert (Eds.), Glow Discharge Plasmas in Analytical Spectroscopy, pp. 15-69. Chichester, West Sussex: John Wiley \& Sons.

Brown, L. S. and G. Gabrielse (1986). Geonium theory: Physics of a single electron or ion in a Penning trap. Reviews of Modern Physics 58(1), 233-311.

Brown, S. C. (1978). A short history of gaseous electronics. In M. Hirsh and H. J. Oskam (Eds.), Gaseous Electronics: Electrical Discharges, Volume 1, pp. 1-18. New York, New York: Academic Press.

Budtz-Jørgensen, C. V. (2001). Studies of Electrical Plasma Discharges. Ph. D. thesis, Aarhus University.

Busch, C. and U. Kortshagen (1995). Numerical solution of the spatially inhomogeneous Boltzmann equation and verification of the nonlocal approach for an argon plasma. Physical Review E 51(1), 280-288.

Cawthron, E. R. (1971). Secondary electron emission from 
solid surfaces bombarded by medium energy ions. Australian Journal of Physics 24(4), 859-870.

Chapin, J. S. (January 1974). The planar magnetron. Research/Development 25(1), 37 - 40.

Cherrington, B. E. (1979). Gaseous electronics and gas lasers. Oxford: Pergamon Press.

Child, C. D. (1911). Discharge from hot CaO. Physical Review 32 (5), 492-511.

Chopra, K. L. (1969). Thin Film Phenomena. New York, New York: McGraw-Hill.

Cobine, J. D. (1958). Gaseous Conductors. New York, New York: Dover Publications.

Coburn, J. W. and W. W. Harrison (1981). Plasma sources in analytical mass spectrometry. Applied Spectroscopy Reviews 17(1), 95-164.

de la Rue, W. and H. W. Müller (1878). Experimental researches on the electric discharge with the chloride of silver battery. Philosophical Transactions of the Royal Society of London 169, 155-241.

de La Rue, W. and H. W. Müller (1880). Experimental researches on the electric discharge with the chloride of silver battery. Philosophical Transaction Royal Society London 171, 65-116.

Delcroix, J.-L. and A. R. Trindade (1974). Gas discharge displays: A critical review. In L. Marton (Ed.), Advances in Electronics and Electron Physics, Volume 35, pp. 87-190. New York, New York: Academic Press.

Den Hartog, E. A., D. A. Doughty, and J. E. Lawler (1988). Laser optogalvanic and fluorescence studies of the cathode region of a glow discharge. Physical Review A 38(5), 24712491.

Depla, D., S. Mahieu, and R. De Gryse (2009). Magnetron sputter deposition: Linking discharge voltage with target properties. Thin Solid Films 517(9), 2825-2839.

Depla, D., S. Mahieu, and J. E. Greene (2010). Sputter deposition processes. In P. M. Martin (Ed.), Handbook of Deposition Technologies for Films and Coatings (3 ed.)., Chapter 5, pp. 253-296. Oxford: William Andrew.

Doughty, D. A., E. A. Den Hartog, and J. E. Lawler (1987). Current balance at the surface of a cold cathode. Physical Review Letters 58(25), 2668-2671.

Doughty, D. K., E. A. Den Hartog, and J. E. Lawler (1985). Optogalvanic measurements of gas temperature in the cathode fall. Applied Physics Letters 46(4), 352-354.

Druyvesteyn, M. J. and F. M. Penning (1940). The mechanism of electrical discharges in gases of low pressure. Reviews of Modern Physics 12(2), 87 - 174.

Duckworth, D. C. and R. K. Marcus (1989). Radio frequency powered glow discharge atomization/ionization source for solids mass spectrometry. Analytical Chemistry 61(17), 1879-1886.

Ecker, G. and O. Zöller (1964). Thermally inhomogeneous plasma column. Physics of Fluids 7(12), 1996-2000.

Eckstein, W. (2007). Sputtering yields. In R. Behrisch and W. Eckstein (Eds.), Sputtering by Particle Bombardment: Experiments and Computer Calculations from Threshold to MeV Energies, Volume 110 of Topics in Applied Physics, pp. 33-187. Berlin Heidelberg: Springer Verlag.

Francis, G. (1956). The glow discharge at low pressure. In S. Flügge (Ed.), Gas Discharges II / Gasentladungen II, Volume 4 of Encyclopedia of Physics, pp. 53-208. Berlin Heidelberg: Springer Verlag.

Fruth, H. F. (1932). Cathode sputtering, A commercial application. Physics 2(4), 280-288.
Garscadden, A. (1978). Ionization waves in glow discharges. In M. Hirsh and H. J. Oskam (Eds.), Gaseous Electronics: Electrical Discharges, Volume 1, pp. 65-107. New York, New York: Academic Press.

Gill, W. D. and E. Kay (1965). Efficient low pressure sputtering in a large inverted magnetron suitable for film synthesis. Review of Scientific Instruments 36(3), 277-282.

Gnaser, H. (2007). Energy and angular distributions of sputtered species. In R. Behrisch and W. Eckstein (Eds.), Sputtering by Particle Bombardment: Experiments and Computer Calculations from Threshold to MeV Energies, Volume 110 of Topics in Applied Physics, Chapter 5, pp. 231328. Berlin Heidelberg: Springer Verlag.

Goebel, D. M. and I. Katz (2008). Fundamentals of Electric Propulsion: Ion and Hall Thrusters. Hoboken, New Jersey: John Wiley \& Sons.

Grimm, W. (1968). Eine neue glimmentladungslampe für die optische emissionsspektralanalyse. Spectrochimica Acta Part B: Atomic Spectroscopy 23(7), 443-454.

Grove, W. R. (1852). On the electro-chemical polarity of gases. Philosophical Magazine, Series 4 4(28), 498-514.

Gudmundsson, J. T., N. Brenning, D. Lundin, and U. Helmersson (2012). The high power impulse magnetron sputtering discharge. Journal of Vacuum Science and Technology A 30(3), 030801.

Harrison, W. W., C. Yang, and E. Oxley (2003). Mass spectrometry of glow discharges. In R. K. Marcus and J. A. C. Broekaert (Eds.), Glow Discharge Plasmas in Analytical Spectroscopy, pp. 71-96. Chichester, West Sussex: John Wiley \& Sons.

Hasselkamp, D. (1992). Kinetic electron emission from solid surfaces under ion bombardment. In D. Hasselkamp, H. Rothard, K.-O. Groeneveld, J. Kemmler, P. Varga, and H. Winter (Eds.), Particle induced electron emission II, Volume 123 of Springer Tracts in Modern Physics, pp. 195. Berlin - Heidelberg: Springer Verlag.

Helmersson, U., M. Lattemann, J. Bohlmark, A. P. Ehiasarian, and J. T. Gudmundsson (2006). Ionized physical vapor deposition (IPVD): A review of technology and applications. Thin Solid Films 513(1-2), 1-24.

Hofer, W. O. (1991). Angular, energy, and mass distribution of sputtered particles. In R. Behrisch and K. Wittmaack (Eds.), Sputtering by Particle Bombardment III: Characteristics of Sputtered Particles, Technical Applications, Volume 64 of Topics in Applied Physics, pp. 15-90. Berlin Heidelberg: Springer Verlag.

Hooper, E. B. (1969). A review of reflex and Penning discharges. In L. Marton and C. Marton (Eds.), Advances in Electronics and Electron Physics, Volume 27, pp. 295-343. New York, New York: Academic Press.

Howatson, A. M. (1976). An Introduction to Gas Discharges (2 ed.). Oxford: Pergamon Press.

Ingold, J. H. (1978). Anatomy of the discharge. In M. Hirsh and H. J. Oskam (Eds.), Gaseous Electronics: Electrical Discharges, Volume 1, pp. 19-64. New York, New York: Academic Press.

Ingold, J. H. (1997). Nonequilibrium positive column. Physical Review E 56(5), 5932-5944.

Kawamura, E. and J. H. Ingold (2001). Particle in cell simulations of low pressure small radius positive column discharges. Journal of Physics D: Applied Physics 34(21), $3150-3162$.

Kay, E. (1962). Impact evaporation (sputtering) and thin film growth in a glow discharge. In L. L. Marton (Ed.), 
Advances of Electronics and Electron Physics, Volume 17, pp. 245-322. New York, New York: Academic Press.

Klawuhn, E., G. C. D'Couto, K. A. A. P. Rymer, M. A. Biberger, and K. B. Levy (2000). Ionized physical-vapor deposition using a hollow-cathode magnetron source for advanced metallization. Journal of Vacuum Science and Technology A 18(4), 1546-1549.

Kolobov, V. I. (2006). Striations in rare gas plasmas. Journal of Physics D: Applied Physics 39(24), R487-R506.

Kolobov, V. I. and L. D. Tsendin (1995). Analytic model of the hollow cathode effect. Plasma Sources Science and Technology 4(4), 551-560.

Langmuir, I. (1913). The effect of space charge and residual gases on thermionic currents in high vacuum. Physical Review 2(6), 450-486.

Lieberman, M. A. and A. J. Lichtenberg (2005). Principles of Plasma Discharges and Materials Processing (2 ed.). New York: John Wiley \& Sons.

Lisovskiy, V. A., K. P. Artushenko, and V. D. Yegorenkov (2016). Child-Langmuir law applicability for a cathode sheath description of glow discharge in hydrogen. Physica Scripta 91(8), 085601.

Lisovskiy, V. A., V. A. Derevianko, and V. D. Yegorenkov (2014). The Child-Langmuir collision laws for the cathode sheath of glow discharge in nitrogen. Vacuum 103, 49-56.

Lisovskiy, V. A., V. A. Derevianko, and V. D. Yegorenkov (2015). DC breakdown in low-pressure $\mathrm{CF}_{4}$. Journal of Physics D: Applied Physics 48(47), 475201.

Lisovskiy, V. A., V. A. Koval, E. P. Artushenko, and V. D. Yegorenkov (2012). Validating the Goldstein-Wehner law for the stratified positive column of dc discharge in an undergraduate laboratory. European Journal of Physics 33(6), 1537-1545.

Lisovskiy, V. A., S. D. Yakovin, and V. D. Yegorenkov (2000). Low-pressure gas breakdown in uniform dc electric field. Journal of Physics D: Applied Physics 33(21), 2722-2730.

Mavrodineanu, R. (1984). Hollow cathode discharges. Journal of Research of the National Bureau of Standards 89(2), 143-185.

Miller, H. C. (1963). Paschen curve in nitrogen. Journal of Applied Physics 34(11), 3418.

Möller, W. and W. Eckstein (1984). TRIDYN - a TRIM simulation code including dynamic composition changes. $\mathrm{Nu}$ clear Instruments and Methods in Physics Research Section B: Beam Interactions with Materials and Atoms 2(1-3), 814-818.

Möller, W., W. Eckstein, and J. Biersack (1988). TRIDYN - binary collision simulation of atomic collisions and dynamic composition changes in solids. Computer Physics Communications 51(3), 355-368.

Mott, N. F. and R. W. Gurney (1948). Electronic Processes in Ionic Crystals (2 ed.). Oxford: Oxford University Press.

Muhl, S. and A. Pérez (2015). The use of hollow cathodes in deposition processes: A critical review. Thin Solid Films 579, 174-198.

Nelis, T. and R. Payling (2003). Glow discharge optical emission spectrometry. In D. J. O'Connor, B. A. Sexton, and R. S. C. Smart (Eds.), Surface Analysis Methods in Materials Science, Volume 23 of Springer Series in Surface Science, pp. 553-559. Berlin Heidelberg: Springer-Verlag.

Oechsner, H. (1975). Sputtering-a review of some recent experimental and theoretical aspects. Applied Physics A: Materials Science \& Processing 8(3), 185-198.

Oleson, N. L. and A. W. Cooper (1968). Moving striations. In
L. Marton and C. Marton (Eds.), Advances in Electronics and Electron Physics, Volume 24, pp. 155-278. New York, New York: Academic Press.

Parilis, E. and L. Kishinevskii (1960). Kinetic secondary electron ejection from molybdenum by cesium ions. Soviet Physics - Solid State 3(4), 885.

Pekarek, L. (1968). Ionization waves (striations) in a discharge plasma. Soviet Physics Uspekhi 11(2), 188-208.

Pekárek, L. and V. Krejčí (1962). Theory of moving striations in plasma of D-C discharge I. Basic equation and its general solution. Czechoslovak Journal of Physics B 12(6), 450460.

Penning, F. M. (1937). Ein neues manometer für niedrige gasdrucke, insbesondere zwischen $10^{-3}$ und $10^{-5} \mathrm{~mm}$. Physica 4(2), 71-75.

Petrov, I., F. Adibi, J. E. Greene, L. Hultman, and J.-E. Sundgren (1993). Average energy deposited per atom: A universal parameter for describing ion-assisted film growth ? Applied Physics Letters 63(1), 36-38.

Phelps, A. V. (2001). Abnormal glow discharges in Ar: experiments and models. Plasma Sources Science and Technology 10(2), 329-343.

Phelps, A. V. and Z. L. Petrović (1999). Cold-cathode discharges and breakdown in argon: surface and gas phase production of secondary electrons. Plasma Sources Science and Technology 8(3), R21-R44.

Phelps, A. V., L. C. Pitchford, C. Pédoussat, and Z. Donkó (1999). Use of secondary-electron yields determined from breakdown data in cathode-fall models for Ar. Plasma Sources Science and Technology 8(4), B1-B2.

Pillow, M. E. (1981). A critical review of spectral and related physical properties of the hollow cathode discharge. Spectrochimica Acta Part B: Atomic Spectroscopy 36(8), 821-843.

Pons-Corbeau, J. (1985). Study of emission and sputtering yields in some alloys and oxide by glow discharge optical spectrometry: Quantification of analysis. Surface and Interface Analysis 7(4), 169-176.

Raizer, Y. P. (1991). Gas Discharge Physics. Berlin: Springer Verlag.

Roth, J. R. (1995). Industrial Plasma Engineering: Principles, Volume 1. Bristol, UK: Institute of Physics Publishing.

Schiller, S., K. Goedicke, J. Reschke, V. Kirchhoff, S. Schneider, and F. Milde (1993). Pulsed magnetron sputter technology. Surface and Coatings Technology 61(1-3), 331 337.

Schoenbach, K. H., M. Moselhy, and W. Shi (2004). Selforganization in cathode boundary layer microdischarges. Plasma Sources Science and Technology 13(1), 177-185.

Schottky, W. (1924). Diffusiontheorie der positiven säule. Zeitschrift für Physik 25(1), 635-640.

Sellers, J. (1998). Asymmetric bipolar pulsed DC: the enabling technology for reactive PVD. Surface and Coatings Technology 98(1-3), 1245 - 1250.

Sigmund, P. (1969). Theory of sputtering. I. Sputtering yield of amorphous and polycrystalline targets. Physical Review 184 (2), 383-416.

Sivoš, J., N. Skoro D Marić, G. Malović, and Z. L. Petrović (2015). Breakdown and dc discharge in low-pressure water vapour. Journal of Physics D: Applied Physics 48(42), 424011.

Stuart, R. V., G. K. Wehner, and G. S. Anderson (1969). Energy distribution of atoms sputtered from polycrystalline 
metals. Journal of Applied Physics 40(2), 803-812.

Thompson, M. W. (1968). The energy spectrum of ejected atoms during the high energy sputtering of gold. Philosophical Magazine 18(152), 377-414.

Thompson, M. W. (1981). Physical mechanisms of sputtering. Physics Reports 69(4), 335-371.

Thornton, J. A. (1978). Magnetron sputtering: basic physics and application to cylindrical magnetrons. Journal of Vacuum Science and Technology 15(2), 171-177.

Thornton, J. A. and J. E. Greene (1994). Sputter deposition processes. In R. F. Bunshah (Ed.), Handbook of Deposition Technologies for Films and Coatings: Science, Technology and Applications (2 ed.)., pp. 275-345. Park Ridge, New Jersey: Noyes Publications.

Thornton, J. A. and A. S. Penfold (1978). Cylindrical magnetron sputtering. In J. L. Vossen and W. Kern (Eds.), Thin Film Processes, Volume 4, pp. 75-113. New York: Academic Press.

Tonks, L. and I. Langmuir (1929). A general theory of the plasma of an arc. Physical Review 34(6), 876-922.

Tsendin, L. D. (1995). Electron kinetics in non-uniform glow discharge plasmas. Plasma Sources Science and Technology 4 (2), 200-211.

Uhrlandt, D. and R. Winkler (1996). Radially inhomogeneous electron kinetics in the DC column plasma. Journal of Physics D: Applied Physics 29(1), 315-320.

Vossen, J. L. and J. J. Cuomo (1978). Glow discharge sputter deposition. In J. L. Vossen and W. Kern (Eds.), Thin Film Processes, Volume 4, pp. 11-73. New York, New York: Academic Press.

Škoro, N., D. Marić, G. Malović, W. G. Graham, and Z. L. Petrović (2011). Electrical breakdown in water vapor. Physical Review E 84(5), 055401.

Škoro, N., D. Marić, and Z. L. Petrović (2008). Effective discharge area of nonequilibrium DC discharges. IEEE Transactions on Plasma Science 36(4), 994-995.

Wagenaars, E., M. D. Bowden, and G. M. W. Kroesen (2005). Plasma emission imaging of a low-pressure argon breakdown. Plasma Sources Science and Technology 14(2), 342350 .

Wagenaars, E., M. D. Bowden, and G. M. W. Kroesen (2007). Measurements of electric-field strengths in ionization fronts during breakdown. Physical Review Letters 98(7), 075002.

Wagenaars, E., N. W. B. Perriëns, W. J. M. Brok, M. D.
Bowden, E. M. van Veldhuizen, and G. M. W. Kroesen (2006). Pre-breakdown light emission phenomena in lowpressure argon between parabolic electrodes. Journal of Physics D: Applied Physics 39(17), 3831-3837.

Waits, R. K. (1978). Planar magnetron sputtering. Journal of Vacuum Science and Technology 15(2), 179 - 187.

Wasa, K. and S. Hayakawa (1969). Low pressure sputtering system of the magnetron type. Review of Scientific Instruments 40(5), 693-697.

Watanabe, S. and N. L. Oleson (1955). Traveling density waves in positive columns. Physical Review 99(6), 17011704.

Weber, L. F. (1985). Plasma displays. In L. E. Tannas (Ed.), Flat-Panel Displays and CRTs, pp. 352-434. New York, New York: Van Nostrand Reinhold.

Westwood, W. D. (1976). Glow discharge sputtering. Progress in Surface Science 7(2), 71-111.

Window, B. and N. Savvides (1986). Charged particle fluxes from planar magnetron sputtering sources. Journal of Vacuum Science and Technology A 4(2), 196-202.

Wright, A. W. (1877a). On a new process for the electrical deposition of metals, and for constructing metal-covered glass specula. American Journal of Science and Arts 14(81), $169-178$

Wright, A. W. (1877b). On the production of transparent metallic films by the electrical discharge in exhausted tubes. American Journal of Science and Arts 14(73), 49-55.

Wright, M. and T. Beardow (1986). Design advances and applications of the rotatable cylindrical magnetron. Journal of Vacuum Science and Technology A 4(3), 388-392.

Yamamura, Y. and H. Tawara (1996). Energy dependence of ion-induced sputtering yields from monoatomic solids at normal incidence. Atomic Data and Nuclear Data Tables 62(2), $149-253$.

Ziegler, J. F., J. P. Biersack, and M. D. Ziegler (2008). SRIMThe Stopping and Range of Ions in Matter. Chester, Maryland: SRIM Co.

Ziegler, J. F., M. D. Ziegler, and J. P. Biersack (2010). SRIM - the stopping and range of ions in matter (2010). Nuclear Instruments and Methods in Physics Research Section B: Beam Interactions with Materials and Atoms 268(11-12), 1818-1823. 